Supporting Information

\title{
Light-Assisted Catalytic Hydrogenation of Carbon Dioxide at a Low Pressure by a Dinuclear Iridium Polyhydride Complex
}

Asuka Takahashi, Masaki Mishima, Kotohiro Nomura, Akiko Inagaki*

Department of Chemistry, Graduate School of Science, Tokyo Metropolitan University, Minami-Osawa 1-1, Hachioji, Tokyo, 192-0397.

\begin{tabular}{|c|c|}
\hline 1. General experimental procedures. & $S 2$ \\
\hline \multicolumn{2}{|l|}{ Figure S1. Experimental setup for the photocatalytic hydrogenation of $\mathrm{CO}_{2}$ by 2 . } \\
\hline 2. Preparation of $\mathbf{A}$, and $\mathbf{B}$ and spectral data of $\mathbf{2}, \mathbf{A}$, and $\mathbf{B}$. & S3 \\
\hline 3. Figure S2. ${ }^{1} \mathrm{H}$ NMR spectrum of 2. & S5 \\
\hline Figure S3. ${ }^{1} \mathrm{H}$ NMR spectrum (hydride region) of 2. & S5 \\
\hline Figure S4. ${ }^{31} \mathrm{P}$ NMR spectrum of 2. & S6 \\
\hline 4. Figure S5. ${ }^{1} \mathrm{H}$ NMR spectrum $\mathbf{A}$. & S6 \\
\hline Figure S6. ${ }^{1} \mathrm{H}$ NMR spectrum of A (hydride region). & $S 7$ \\
\hline Figure S7. ${ }^{31} \mathrm{P}$ NMR spectra of $\mathbf{A}$ & $S 7$ \\
\hline Figure S8. ${ }^{13} \mathrm{C}$ NMR spectrum of $\mathbf{A}$. & $S 8$ \\
\hline 5. Figure S9. ${ }^{1} \mathrm{H}$ NMR spectra of $\mathbf{B}$. & $S 9$ \\
\hline Figure S10. ${ }^{1} \mathrm{H}$ NMR spectrum of $\mathbf{B}$ (hydride region). & $S 9$ \\
\hline Figure S11. ${ }^{31} \mathrm{P}$ NMR spectra of $\mathbf{B}$. & $S 10$ \\
\hline Figure S12. ${ }^{13} \mathrm{C}$ NMR spectra of $\mathbf{B}$. & $S 10$ \\
\hline 6. Figure S13 and $14 \cdot{ }^{13} \mathrm{C}-{ }^{1} \mathrm{H}$ HSQC NMR spectra of A and $\mathbf{B}$. & $S 11$ \\
\hline 7. Figures S15 and S16. ESI-MS spectral data for $\mathbf{A}$ and $\mathbf{B}$. & $S 13$ \\
\hline 8. Figure S17. UV-vis absorption spectra of $\mathbf{2}, \mathbf{A}$ ( and $\mathbf{A}^{\prime}$ ), and $\mathbf{B}$ (and $\mathbf{B}^{\prime}$ ) & $S 14$ \\
\hline $\begin{array}{l}\text { 9. Figure S18. }{ }^{1} \mathrm{H} \text { and }{ }^{31} \mathrm{P} \text { NMR spectra of } 2 \text { in the H-D exchange reaction under } \\
\text { irradiation }(395 \mathrm{~nm}) \text { and dark condition. }\end{array}$ & $S 15$ \\
\hline $\begin{array}{l}\text { 10. Figure S19. ESI-MS spectra of } 2 \text { after H-D exchange reaction under irradiated ( } 395 \\
\mathrm{~nm} \text { ) condition. }\end{array}$ & $S 16$ \\
\hline 11. Figure S20. Frontier orbitals of 2. & $S 17$ \\
\hline 12. Figure S21. Preliminary TDDFT calculation results for 2. & S18 \\
\hline 13. X-ray Structural Determinations and ORTEP diagram of B-OMe (Figure S22). & S19 \\
\hline 14. Table S1. Crystal data and structure refinement for B-OMe. & $S 20$ \\
\hline $\begin{array}{l}\text { 15. Table S2. Atomic coordinates (x 104) and equivalent isotropic displacement } \\
\text { parameters }\left(\AA^{2} \times 10^{3}\right) \text { for B-OMe. }\end{array}$ & $S 21$ \\
\hline 16. Table S3. Bond lengths $[\AA]$ and angles $\left[^{\circ}\right]$ for B-OMe. & $S 25$ \\
\hline
\end{tabular}




\section{Experimental Procedures}

General. All experiments were carried out under a nitrogen atmosphere using standard Schlenk techniques. All solvents were treated with appropriate drying agents, distilled, and stored under nitrogen atmosphere. All the chemicals were purchased from common commercial suppliers. ${ }^{1} \mathrm{H}$ (400 $\mathrm{MHz})$ and ${ }^{31} \mathrm{P}(162 \mathrm{MHz})$ NMR spectra were acquired on a JEOL JNM-AL400 FT-NMR spectrometers. ${ }^{13} \mathrm{C}$ NMR spectra were acquired on a Bruker AVANCE III 600 FT-NMR spectrometers. Solvents for NMR measurements $\left(\left(\mathrm{CD}_{3}\right)_{2} \mathrm{CO}\right)$ were dried over anhydrous $\mathrm{KOH}$ or molecular sieves, degassed, and stored under $\mathrm{N}_{2}$ atmosphere. UV-vis absorption was obtained on a JASCO V-670 spectrometer, respectively. Electrospray ionization (ESI) mass spectra were recorded on a Bruker MicroTOF II mass spectrometers.

Standard procedure for catalytic hydrogenation of carbon dioxide. Catalyst $(0.25-2.0 \mu \mathrm{mol})$ and base $(2.3 \mathrm{mmol})$ are dissolved in solvents $(3 \mathrm{~mL})$ set to an appropriate concentration. 3(trimethylsilyl)-1-propanesulfonate is added to the reaction mixture as an internal standard. To the 10 $\mathrm{mL}$ glass autoclave, the solution was charged under $\mathrm{H}_{2}$ atmosphere and pressurized by $\mathrm{H}_{2}(5 \mathrm{~atm})$ and then by $\mathrm{CO}_{2}(5 \mathrm{~atm})$ to make the condition pressurized to 10 atm in $1: 1$ gas ratio of $\mathrm{CO}_{2}$ and $\mathrm{H}_{2}$. (Caution! The total pressure of the glass autoclave should be kept under the suitable and specified pressure level of each container.) The reaction mixture was stirred under irradiation by $3 \mathrm{~W}$ LED lamp $(\lambda=395 \mathrm{~nm})$ for $24 \mathrm{~h}$. After the reaction, the solution was dried under reduced pressure and the obtained solid was analyzed by ${ }^{1} \mathrm{H}$ NMR in $\mathrm{D}_{2} \mathrm{O}$ to calculate the yield (Figure $\mathrm{S} 1$ ).

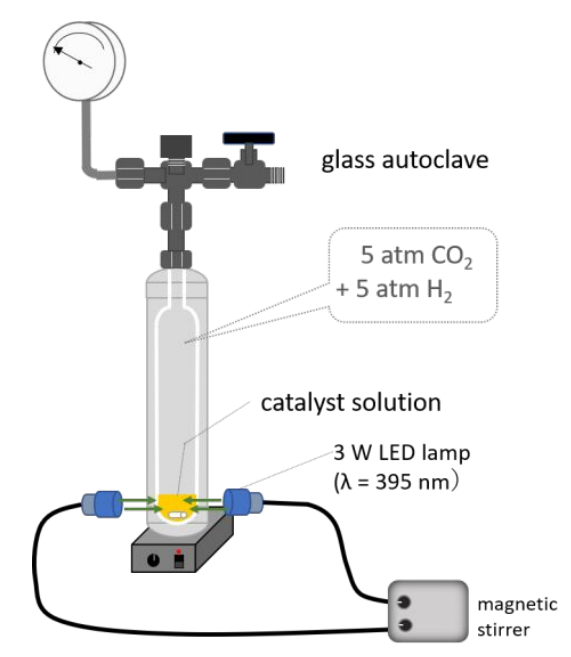

Figure S1. Experimental setup for the photocatalytic hydrogenation of $\mathrm{CO}_{2}$ by 2 . 


\section{Preparation and spectral data of 2.}

A methanol $(2.40 \mathrm{~mL})$ solution of $3(36.8 \mathrm{mg}, 0.0399 \mathrm{mmol})$ was degassed by freeze-pump-thaw method, and then 1atm of $\mathrm{H}_{2}$ gas was introduced. The solution became cloudy after stirring the solution for $4 \mathrm{~h}$, and $\mathrm{Et}_{2} \mathrm{O}$ was added for precipitation. The obtained orange powder was washed with $\mathrm{Et}_{2} \mathrm{O}$ at $0^{\circ} \mathrm{C}$ and dried under vacuum to afford 2 as orange powder $(28.7 \mathrm{mg}, 0.0186 \mathrm{mmol}, 93.0 \%)$.

\section{Preparation and spectral data of monoformate $A$ (mixture of stereoisomers $A$ and A').}

To an acetone $(2.0 \mathrm{~mL})$ solution of $2(54.3 \mathrm{mg}, 0.0351 \mathrm{mmol})$, HCOOH $(13.5 \mu \mathrm{L}, 0.358 \mathrm{mmol})$ was added. After stirring the solution for $6 \mathrm{~h}, n$-hexane was added to isolate $\mathbf{A}$ as an orange powder (43.8 $\mathrm{mg}, 0.0276 \mathrm{mmol}, 78.4 \%)$.

${ }^{1} \mathrm{H}$ NMR (400 MHz, acetone- $\left.d_{6}, \delta / \mathrm{ppm}\right) 8.20(\mathrm{dd}, J=10.6,7.8 \mathrm{~Hz}, 4 \mathrm{H}), 8.08(\mathrm{dd}, J=7.6,4.4 \mathrm{~Hz}$, $4 \mathrm{H}), 8.03-7.94(\mathrm{~m}, 4 \mathrm{H}), 7.87(\mathrm{~d}, J=5.6 \mathrm{~Hz}, 2 \mathrm{H}$, formate- $H)$, 7.74-7.08 (m, $76 \mathrm{H}), 6.96-6.89(\mathrm{~m}, 6$ H), $6.85(\mathrm{t}, J=7.2 \mathrm{~Hz}, 4 \mathrm{H}), 6.65(\mathrm{~m}, 6 \mathrm{H}), 6.25(\mathrm{t}, J=8.0 \mathrm{~Hz}, 2 \mathrm{H}), 5.77(\mathrm{~d}, J=8.8 \mathrm{~Hz}, 2 \mathrm{H}), 5.56$ $(\mathrm{d}, J=8.0 \mathrm{~Hz}, 2 \mathrm{H}),-5.30\left(\mathrm{~d}, J=69.2 \mathrm{~Hz}, 2 \mathrm{H}, \mathbf{A}\right.$ or $\mathbf{A}^{\prime}$, bridged H), $-8.81\left(\mathrm{t}, J=63.2 \mathrm{~Hz}, 2 \mathrm{H}, \mathbf{A}^{\prime}\right.$ or A, bridged H), $-18.2\left(\mathrm{q}, J=21.8 \mathrm{~Hz}, 2 \mathrm{H}, \mathbf{A}\right.$ or $\mathbf{A}^{\prime}$, terminal $\left.\mathrm{H}\right),-21.2\left(\mathrm{t}, J=14.9 \mathrm{~Hz}, 2 \mathrm{H}, \mathbf{A}^{\prime}\right.$ or $\mathbf{A}$, terminal $\mathrm{H}$ )

${ }^{31} \mathrm{P}\left\{{ }^{1} \mathrm{H}\right\}$ NMR (161.8 MHz, acetone- $\left.d_{6}, \delta / \mathrm{ppm}\right)-8.04$ (brs), -14.0 (brs).

${ }^{13} \mathrm{C}\left\{{ }^{1} \mathrm{H}\right\}$ NMR (100 MHz, acetone- $\left.d_{6}, \delta / \mathrm{ppm}\right) 178.1$ (s, HCOO), 141.7 (brs, 1), 139.9-139.4 (m, 6), 136.1 (brs, 9), 135.7-135.1 (br, 9), 134.5 (brs,9), 134.0 (brs, 9), 133.4-131.8 (brs, 11), 130.7 (brs, 3 or 8), 130.0-128.4 (m, 3, 8, 10), 128.1 (brs, 10), 127.4, 127.2, 126.9, 126.7, 126.4 (s, 4, 2), 121.3, 121.0, 120.9, 120.8 (s, 5), 121.3 (brs, 5), 69.0 (7), $54.4\left(\mathrm{t}, J_{\mathrm{CP}}=76 \mathrm{~Hz}, 7\right)$.

Anal. Calcd. for $\mathrm{C}_{75} \mathrm{H}_{61} \mathrm{Ir}_{2} \mathrm{O}_{2} \mathrm{P}_{4} \mathrm{BF}_{4} \cdot 2 \mathrm{FA}$ : C, 55.00; H, 3.90. Found: C, 54.76; H, 4.01.

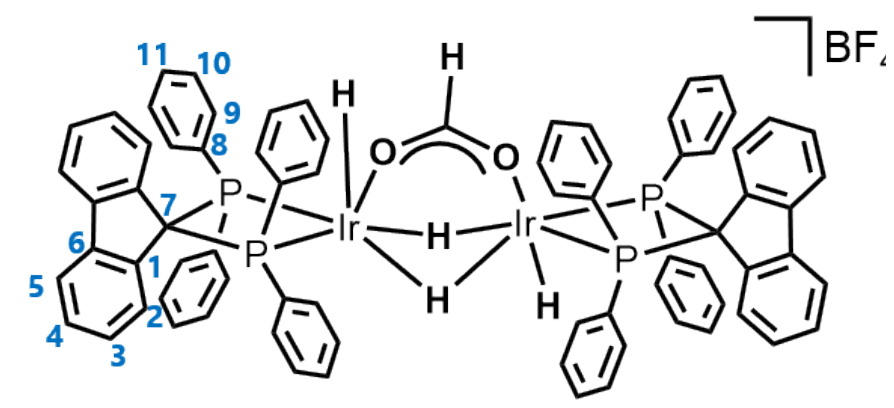

Preparation and spectral data of bisformate B (mixture of stereoisomers B and B').

To an acetone $(2.0 \mathrm{~mL})$ solution of $2(40.1 \mathrm{mg}, 0.0259 \mathrm{mmol})$, HCOOH $(10.0 \mu \mathrm{L}, 0.265 \mathrm{mmol})$ was 
added. After stirring the solution for $7 \mathrm{~h}$ with irradiation (395 $\mathrm{nm}$ LED light), $n$-hexane was added to isolate $\mathbf{B}$ as an orange powder $(27.2 \mathrm{mg}, 0.0166 \mathrm{mmol}, 64.2 \%)$.

${ }^{1} \mathrm{H}$ NMR (400 MHz, acetone- $\left.d_{6}, \delta / \mathrm{ppm}\right) 9.02(\mathrm{t}, J=9.8 \mathrm{~Hz}), 8.95$ (d, $J=2.8 \mathrm{~Hz}, 1 \mathrm{H}$, formate- $H$ ), $8.93(\mathrm{~d}, J=2.8 \mathrm{~Hz}, 1 \mathrm{H}$, formate- $H), 8.89(\mathrm{~m}, J=2.8 \mathrm{~Hz}), 8.14(\mathrm{dd}, J=8.0,7.6 \mathrm{~Hz}), 7.93(\mathrm{dd}, J=8.0$, $7.6 \mathrm{~Hz}$ ), 7.81 (br, m), 7.65 (dd, $J=8.0,7.6 \mathrm{~Hz}), 7.52-7.11(\mathrm{~m}), 6.90$ (t, $4 \mathrm{H}, J=7.2 \mathrm{~Hz}$ ), 6.73-6.59 (m), $6.54(\mathrm{~m}, J=7.4 \mathrm{~Hz}), 6.45(\mathrm{td}, J=8.0,2.9 \mathrm{~Hz}), 6.37$ (d, $J=8.0 \mathrm{~Hz}), 5.55(\mathrm{~d}, J=8.0 \mathrm{~Hz}),-13.5$ (t, $J=59.0 \mathrm{~Hz}, 1 \mathrm{H}, \mathbf{B}$ and B' bridged H), -20.6 (brs, $2 \mathrm{H}, \mathbf{B}$ or B', terminal H), -21.6 (brs, $2 \mathrm{H}, \mathbf{B}^{\prime}$ or $\mathbf{B}$, terminal $\mathbf{H}$ ). Since above data is a mixture of $\mathbf{B}$ and $\mathbf{B}$ ' in approximately $2: 1$ ratio. Some peaks are overlapped and could not assign each peak to which isomer. Thus, integral intensity is not included here.

${ }^{31} \mathrm{P}\left\{{ }^{1} \mathrm{H}\right\}$ NMR (161.8 MHz, acetone- $\left.d_{6}, \delta / \mathrm{ppm}\right)-13.8(\mathrm{br}, \mathrm{m}),-18.7(\mathrm{dd}, J=25,18 \mathrm{~Hz})$.

${ }^{13} \mathrm{C}\left\{{ }^{1} \mathrm{H}\right\}$ NMR (100 MHz, acetone- $\left.d_{6}, \delta / \mathrm{ppm}\right) 180.1$ (s, HCOO), 143.0, 141.3 (brs, 1), 139.7 (s, 6), 136.1-134.7 (m, 9), 132.6, 132.4, 132.1, 131.8 (s, 11), 130.6, 130.5 (s, 3, 8), 129.7 (s), 129.2-128.2 (m, PAr), 127.3 (s, 4), 126.6(s, 2), 121.5 (s, 5), 120.9 (s, 5), 69.0(7), 54.4 (m, 7).

Anal. Calcd. for $\mathrm{C}_{76} \mathrm{H}_{61} \mathrm{BF}_{4} \mathrm{Ir}_{2} \mathrm{O}_{4} \mathrm{P}_{4} \cdot 5 \mathrm{FA}$ : C, 52.21; H, 3.84. Found: C, 52.45; H, 4.01.

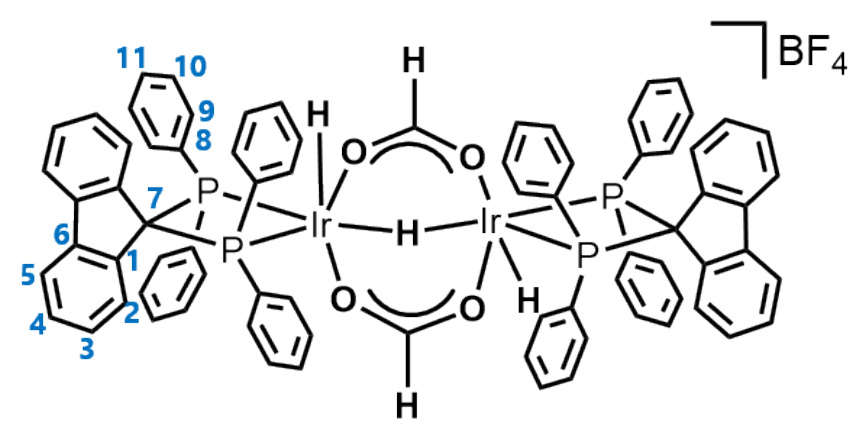



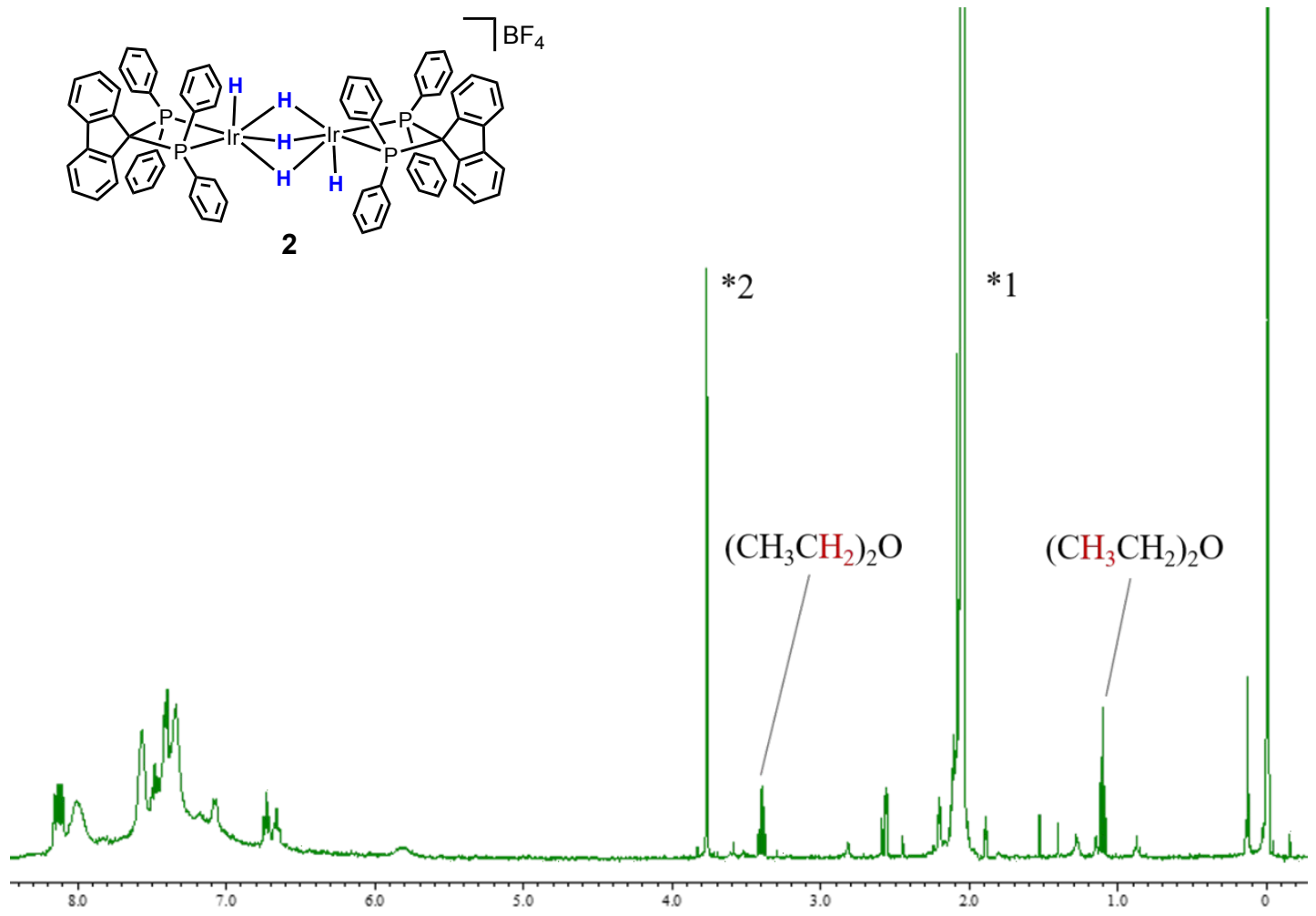

*1 residual proton signal of $\left(\mathrm{CD}_{3}\right)_{2} \mathrm{CO}$

$* 2$ impurity in $\left(\mathrm{CD}_{3}\right)_{2} \mathrm{CO}$

Figure S2. ${ }^{1} \mathrm{H}$ NMR spectra of $2\left(400 \mathrm{MHz}\right.$, acetone- $d_{6}$, r.t.)

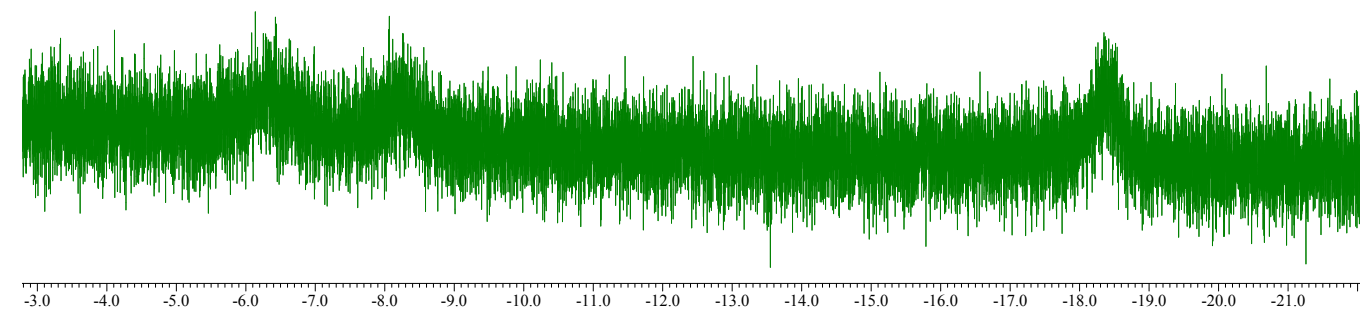

Figure S3. ${ }^{1} \mathrm{H}$ NMR spectra (hydride region) of hydride region of 2 (400 MHz, acetone- $d_{6}$, r.t.). 


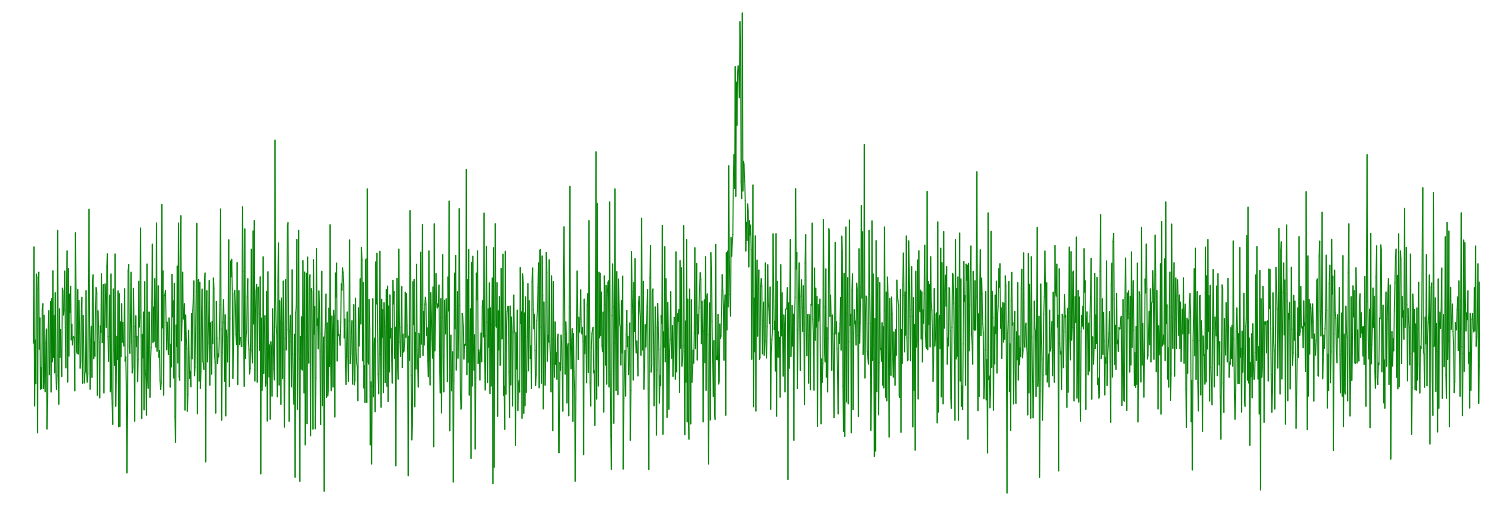

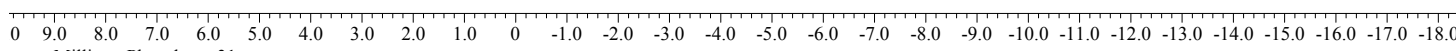

Figure S4. ${ }^{31} \mathrm{P}$ NMR spectra of 2 (161.8 MHz, acetone- $d_{6}$, r.t.).

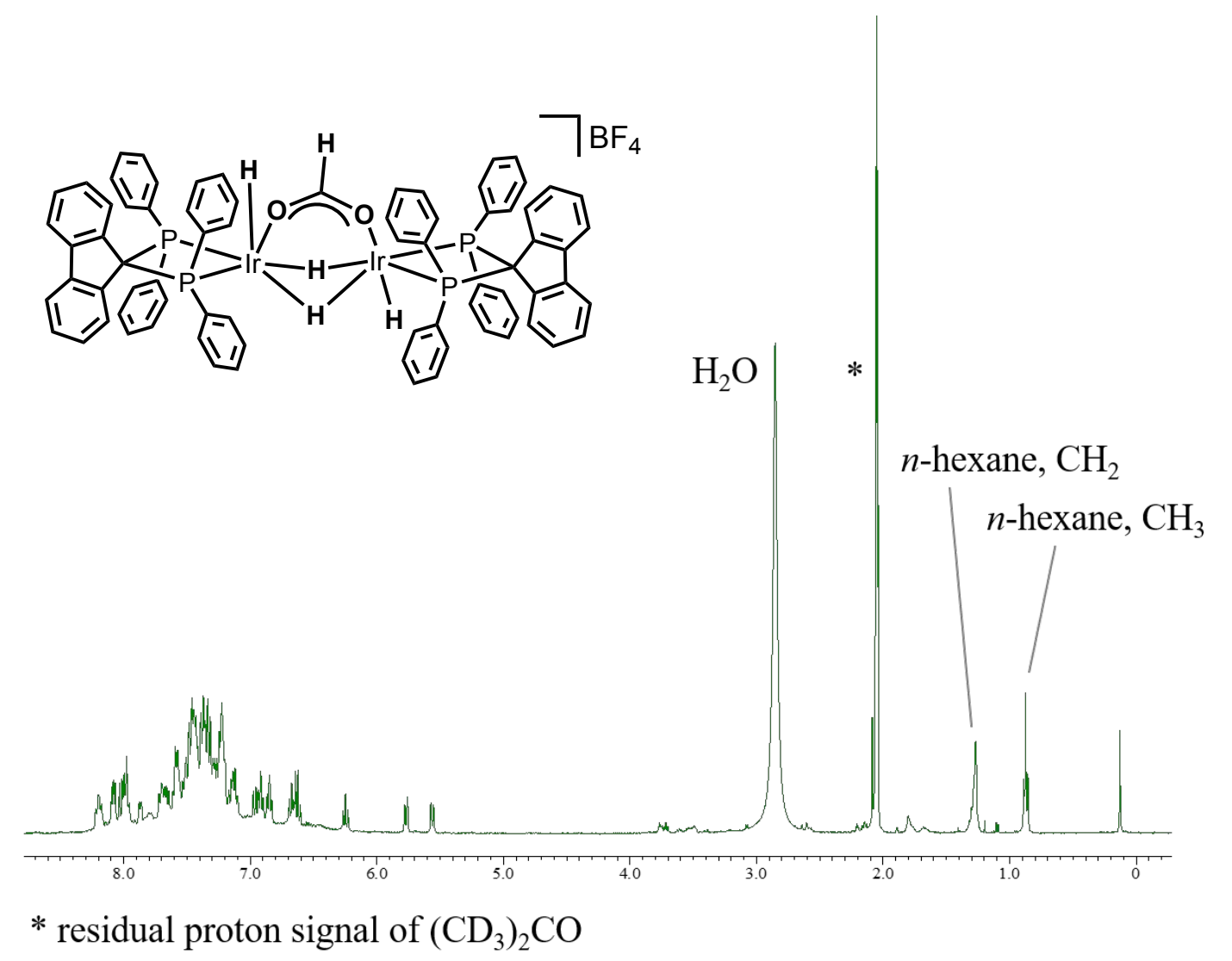

Figure S5. ${ }^{1} \mathrm{H}$ NMR spectra of $\mathbf{A}\left(400 \mathrm{MHz}\right.$, acetone- $d_{6}$, r.t.). 


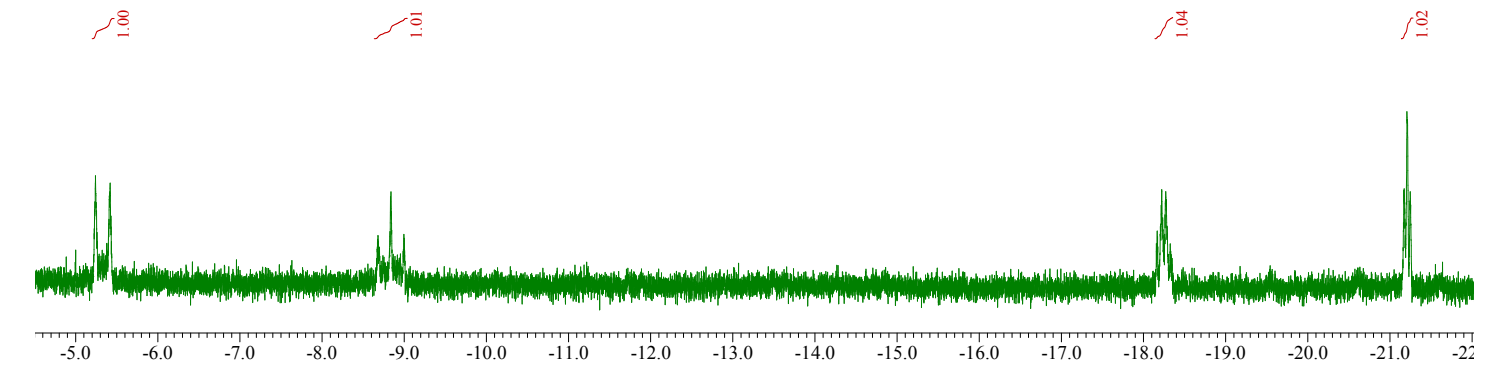

Figure S6. ${ }^{1} \mathrm{H}$ NMR spectra of hydride region of $\mathbf{A}\left(400 \mathrm{MHz}\right.$, acetone- $d_{6}$, r.t.).

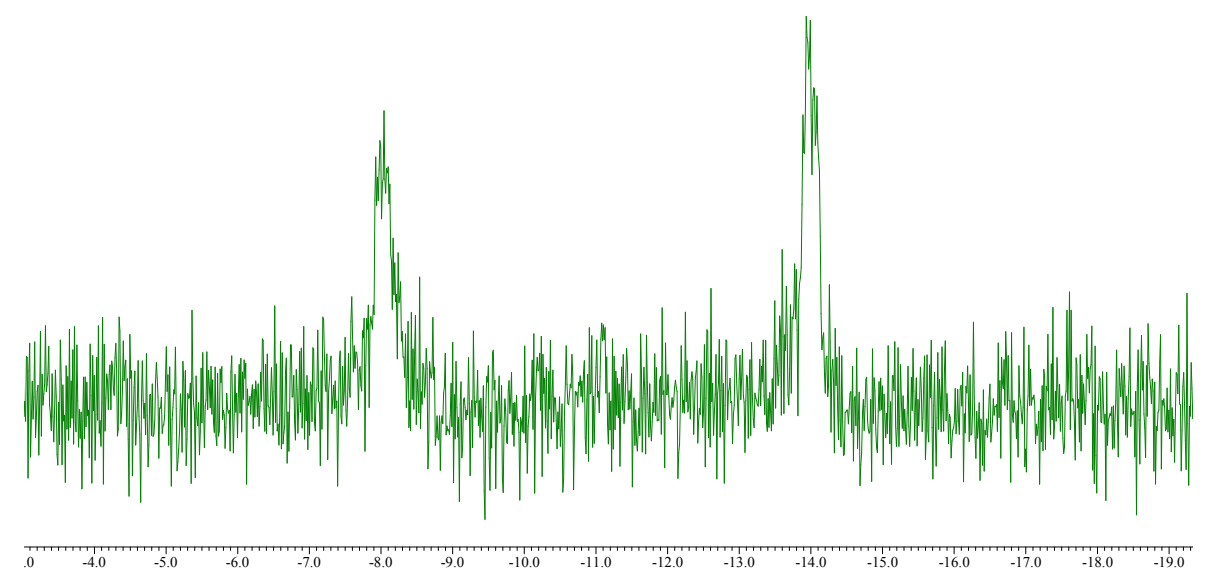

Figure S7 ${ }^{31} \mathrm{P}$ NMR spectra of $\mathbf{A}\left(161.8 \mathrm{MHz}\right.$, acetone- $d_{6}$, r.t.). 

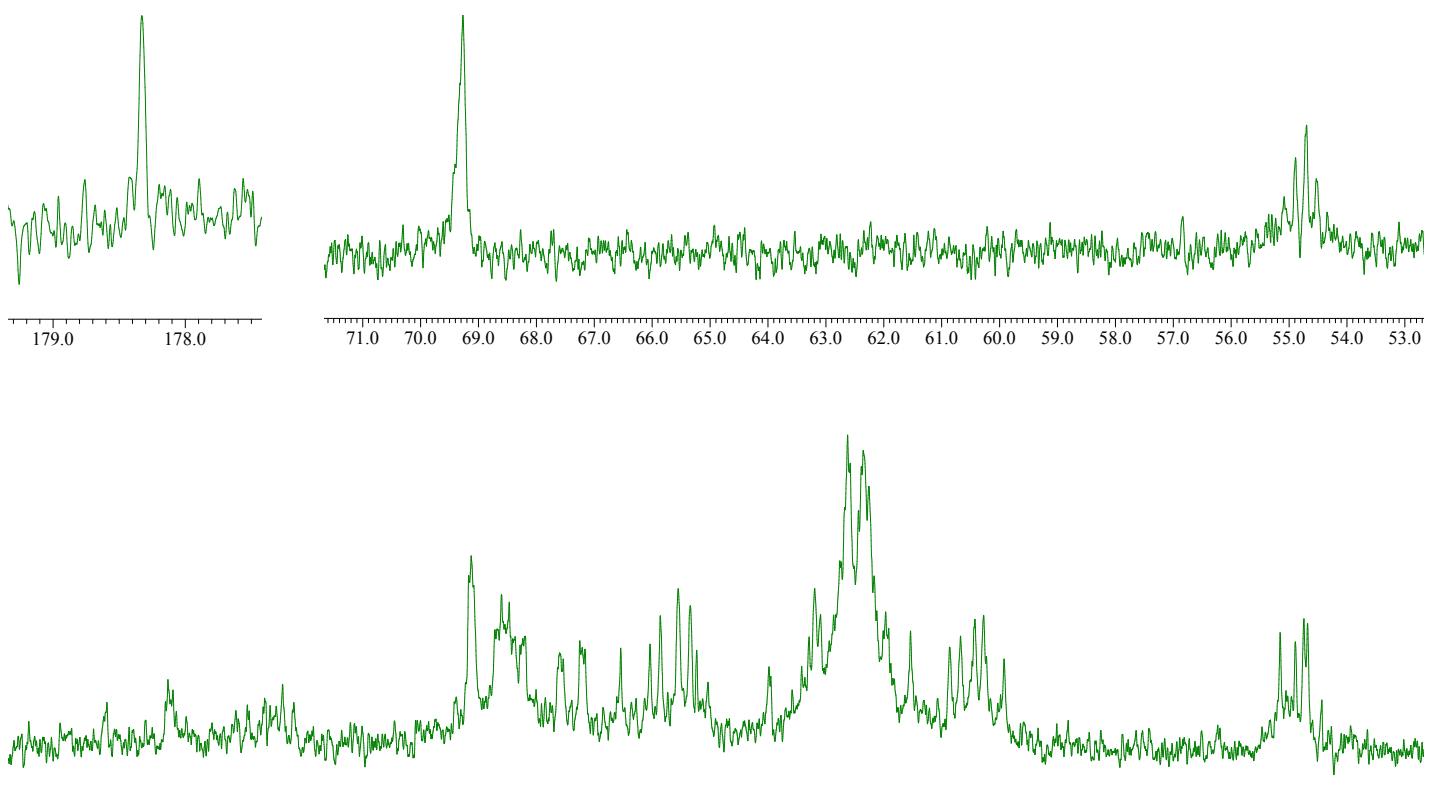

\begin{tabular}{lllllllllllllllllllllllllllllllllllllllllllll}
\hline 0 & 144.0 & 143.0 & 142.0 & 141.0 & 140.0 & 139.0 & 138.0 & 137.0 & 136.0 & 135.0 & 134.0 & 133.0 & 132.0 & 131.0 & 130.0 & 129.0 & 128.0 & 127.0 & 126.0 & 125.0 & 124.0 & 123.0 & 122.0 & 121.0 & 120.0 & 115
\end{tabular}

Figure S8 ${ }^{13} \mathrm{C}$ NMR spectra of $\mathbf{A}\left(125 \mathrm{MHz}\right.$, acetone- $d_{6}$, r.t.). 


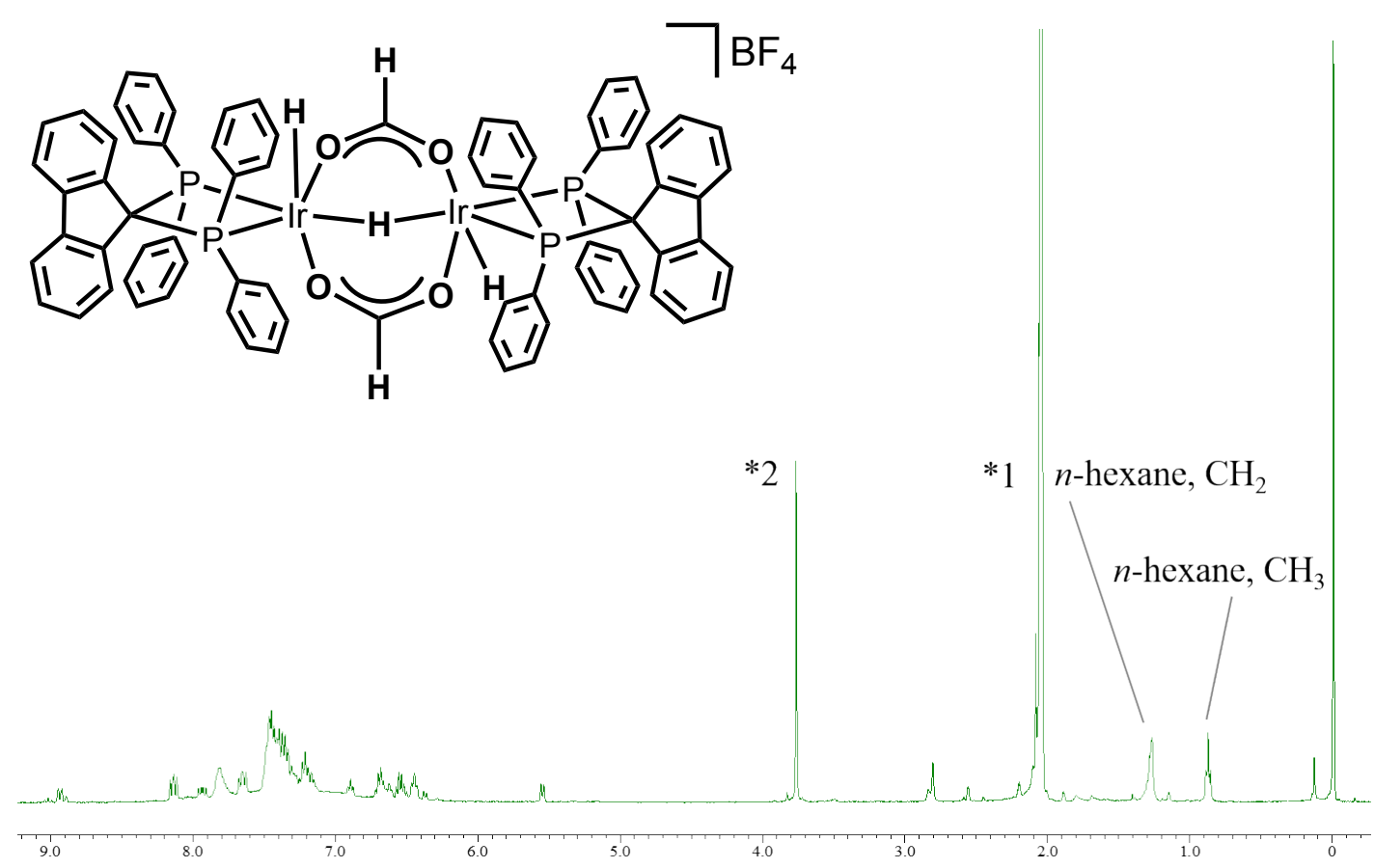

*1 residual proton signal of $\left(\mathrm{CD}_{3}\right)_{2} \mathrm{CO}$

*2 impurity in $\left(\mathrm{CD}_{3}\right)_{2} \mathrm{CO}$

Figure S9. ${ }^{1} \mathrm{H}$ NMR spectra of $\mathbf{B}\left(400 \mathrm{MHz}\right.$, acetone- $d_{6}$, r.t.).

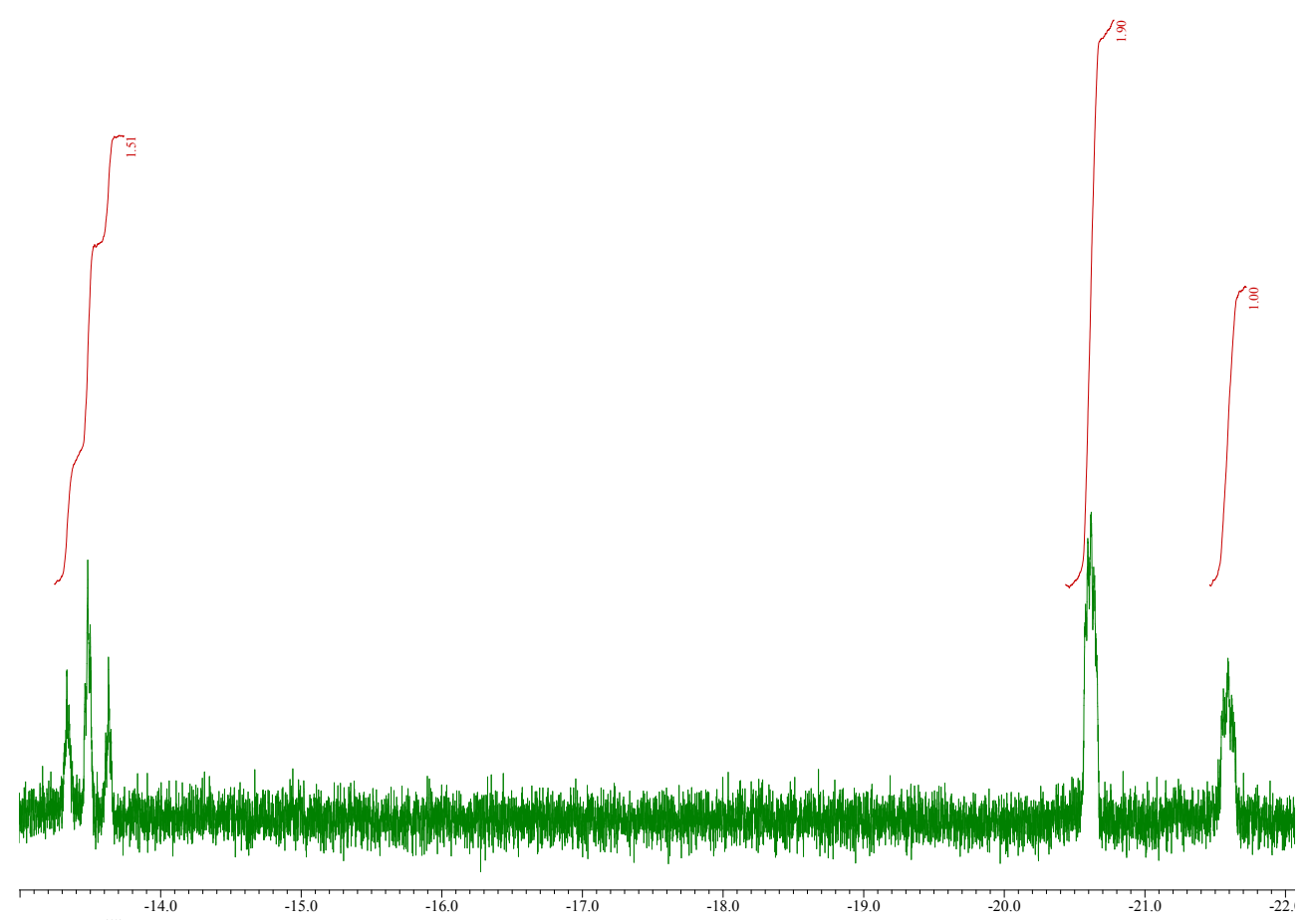

Figure S10. ${ }^{1} \mathrm{H}$ NMR spectra of hydride region of $\mathbf{B}\left(400 \mathrm{MHz}\right.$, acetone- $d_{6}$, r.t.). 


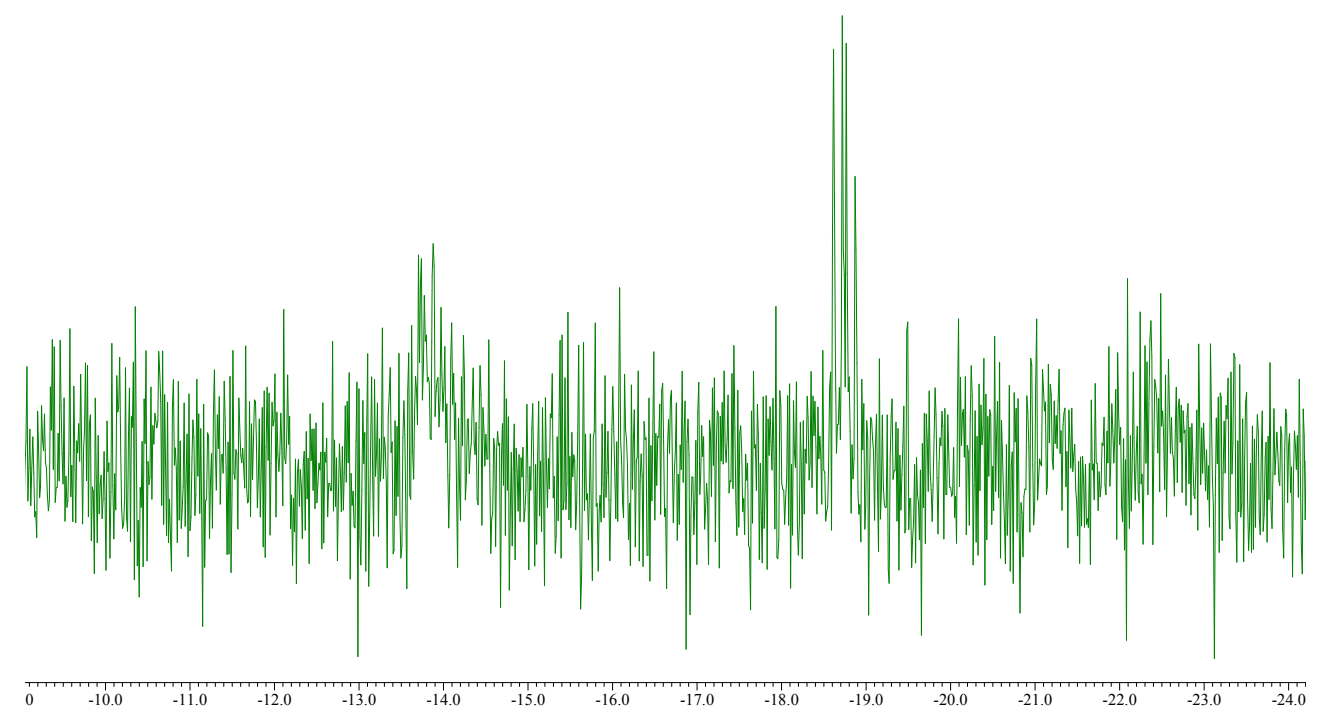

Figure S11. ${ }^{31} \mathrm{P}$ NMR spectra of $\mathbf{B}\left(161.8 \mathrm{MHz}\right.$, acerone- $d_{6}$, r.t.).
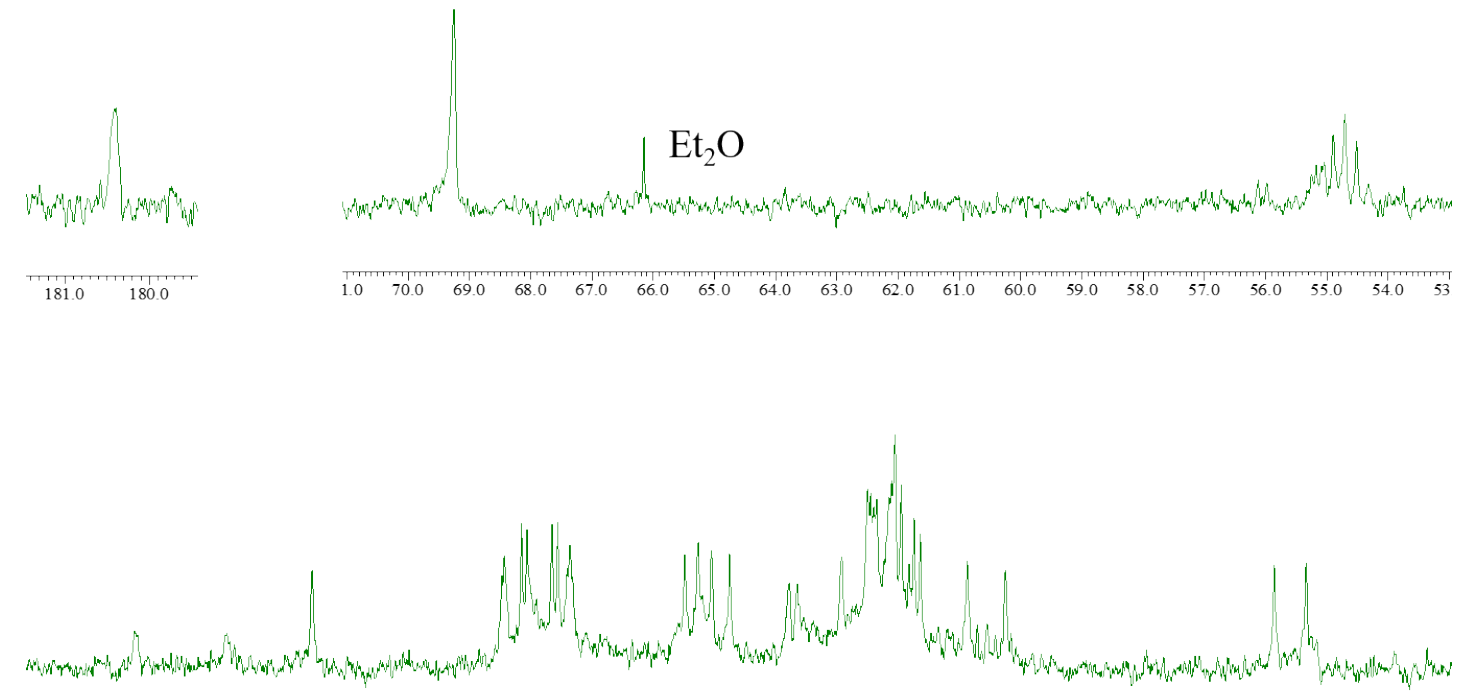

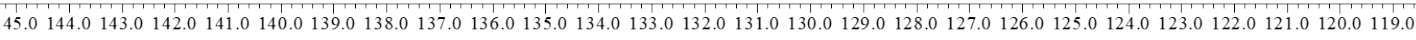

Figure S12. ${ }^{13} \mathrm{C}$ NMR spectra of $\mathbf{B}\left(125 \mathrm{MHz}\right.$, acetone- $d_{6}$, r.t.). 


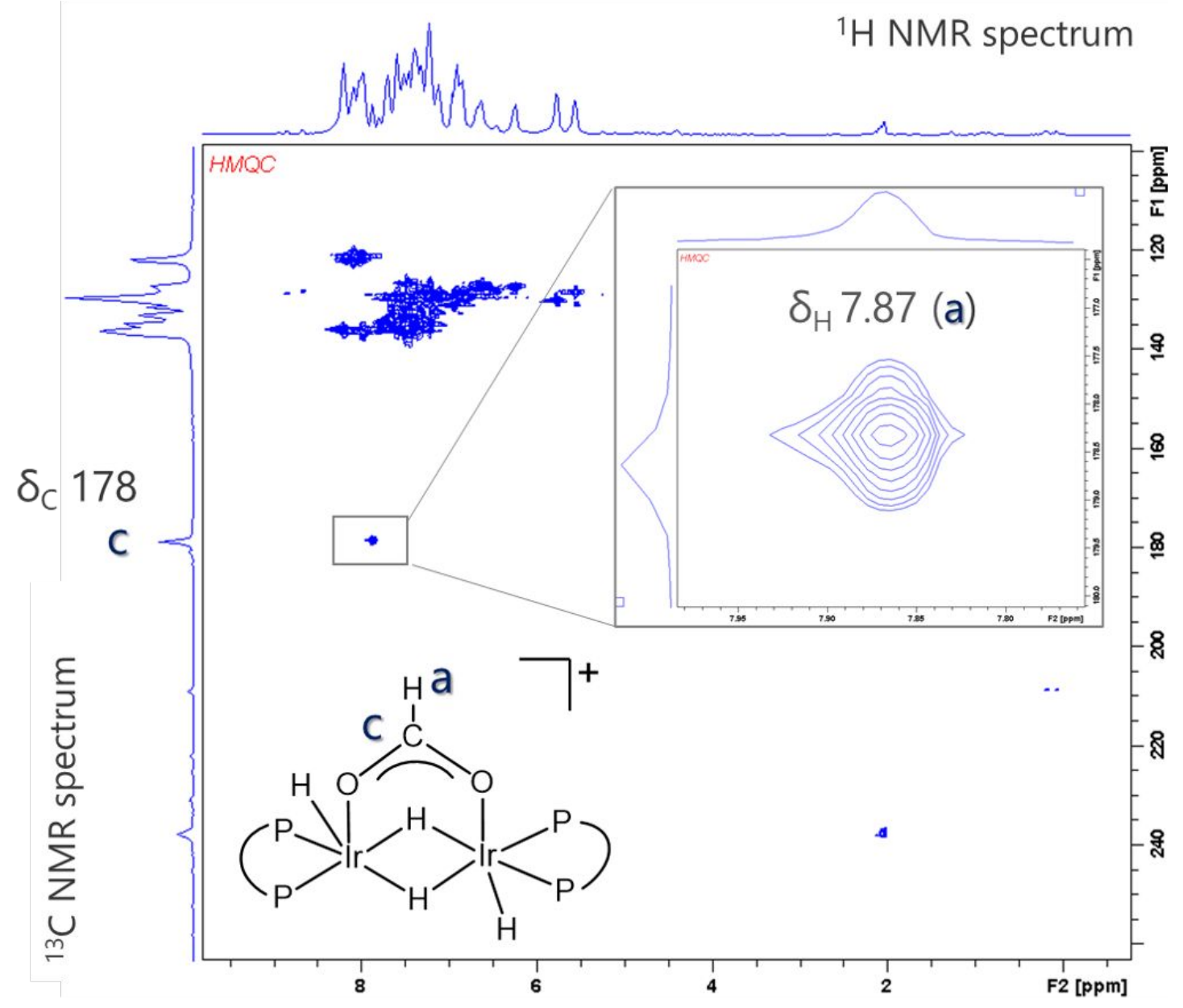

Figure S13. ${ }^{1} \mathrm{H},{ }^{13} \mathrm{C}$-HSQC spectra of intermediate A $\left(20^{\circ} \mathrm{C}\right.$, acetone- $\left.d_{6}\right)$ 


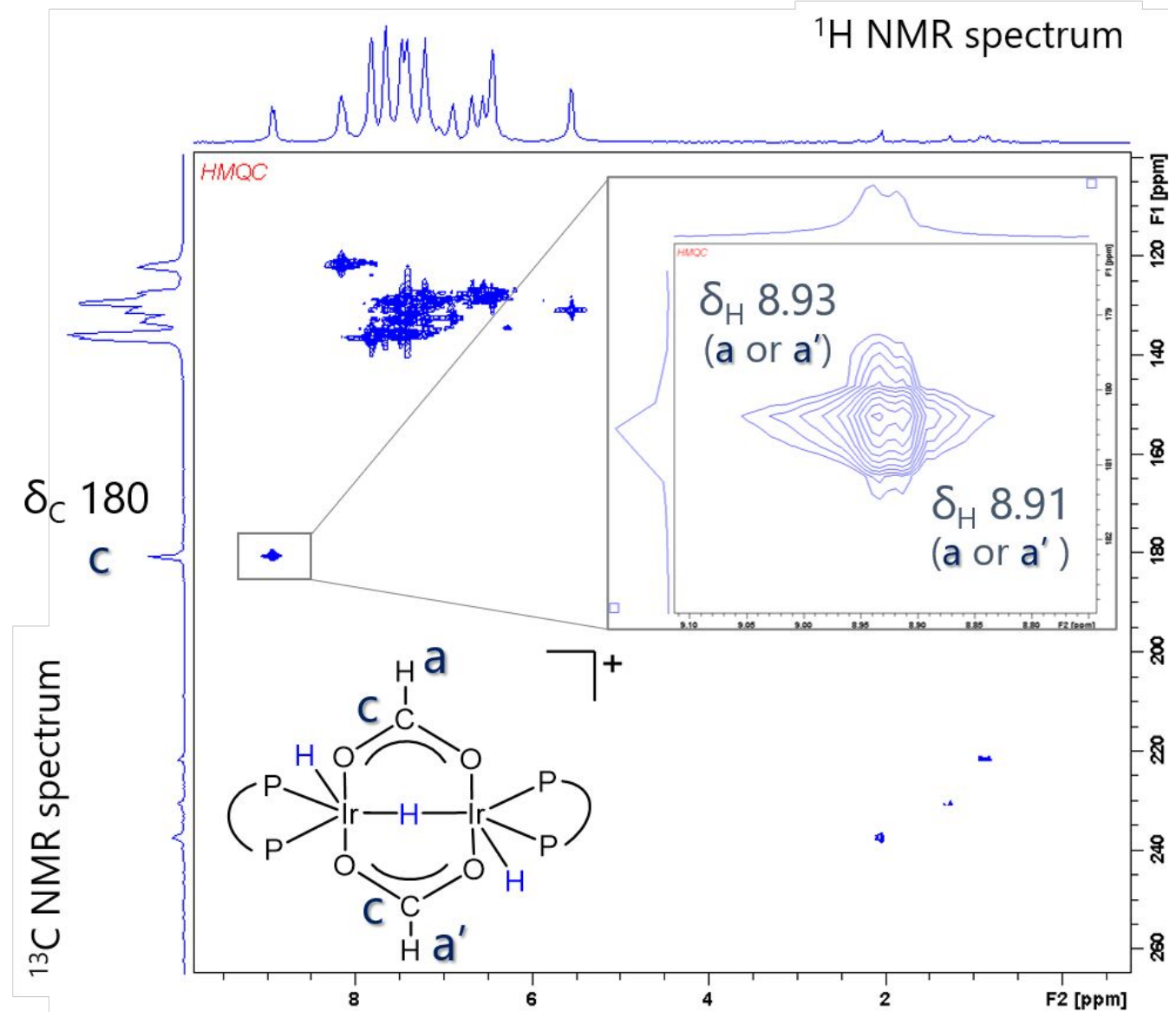

Figure S14. ${ }^{1} \mathrm{H},{ }^{13} \mathrm{C}-\mathrm{HMQC}$ spectra of intermediate $\mathbf{B}\left(20^{\circ} \mathrm{C}\right.$, acetone- $\left.d_{6}\right)$ 


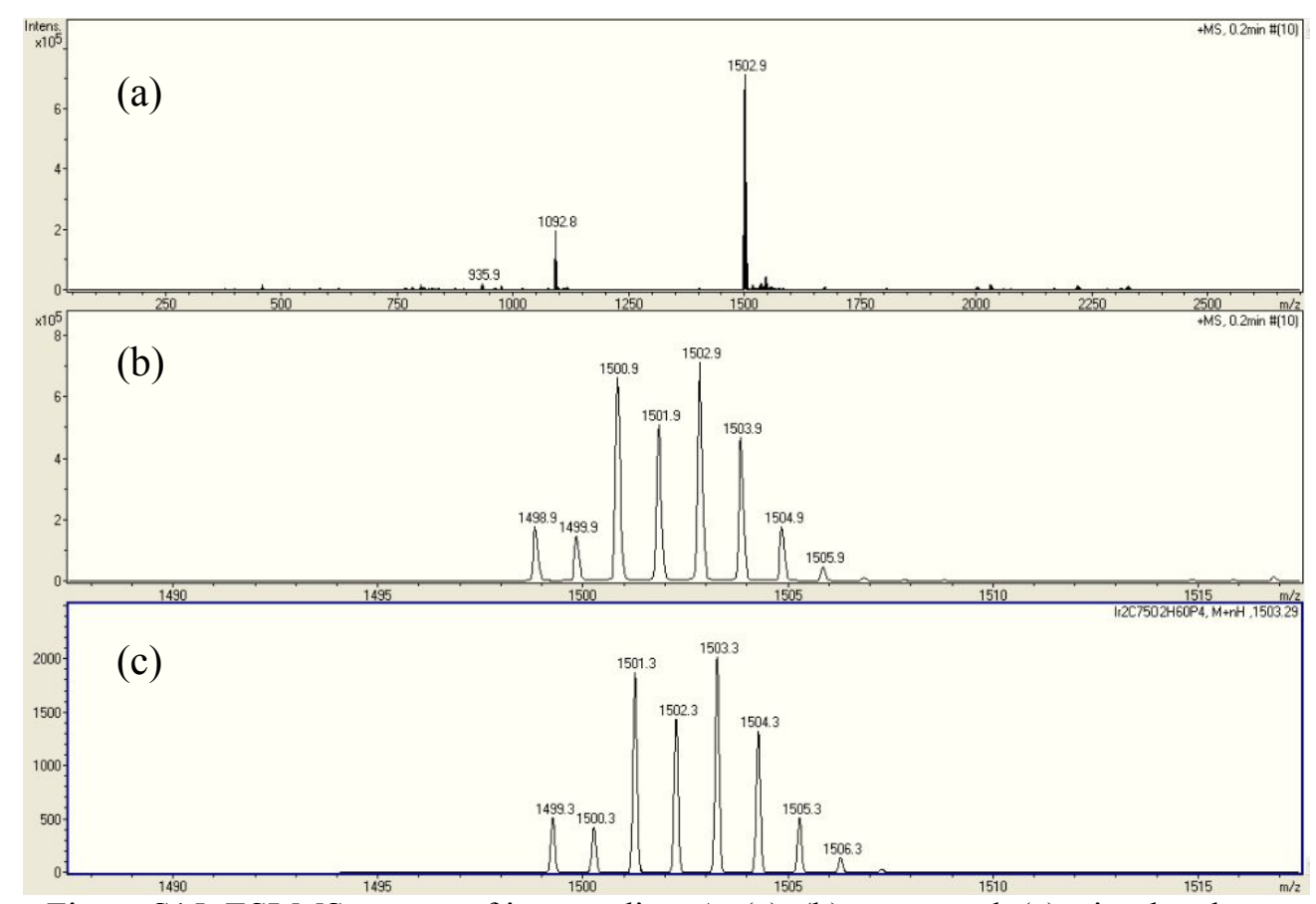

Figure S15. ESI-MS spectra of intermediate A. (a), (b); measured. (c); simulated.

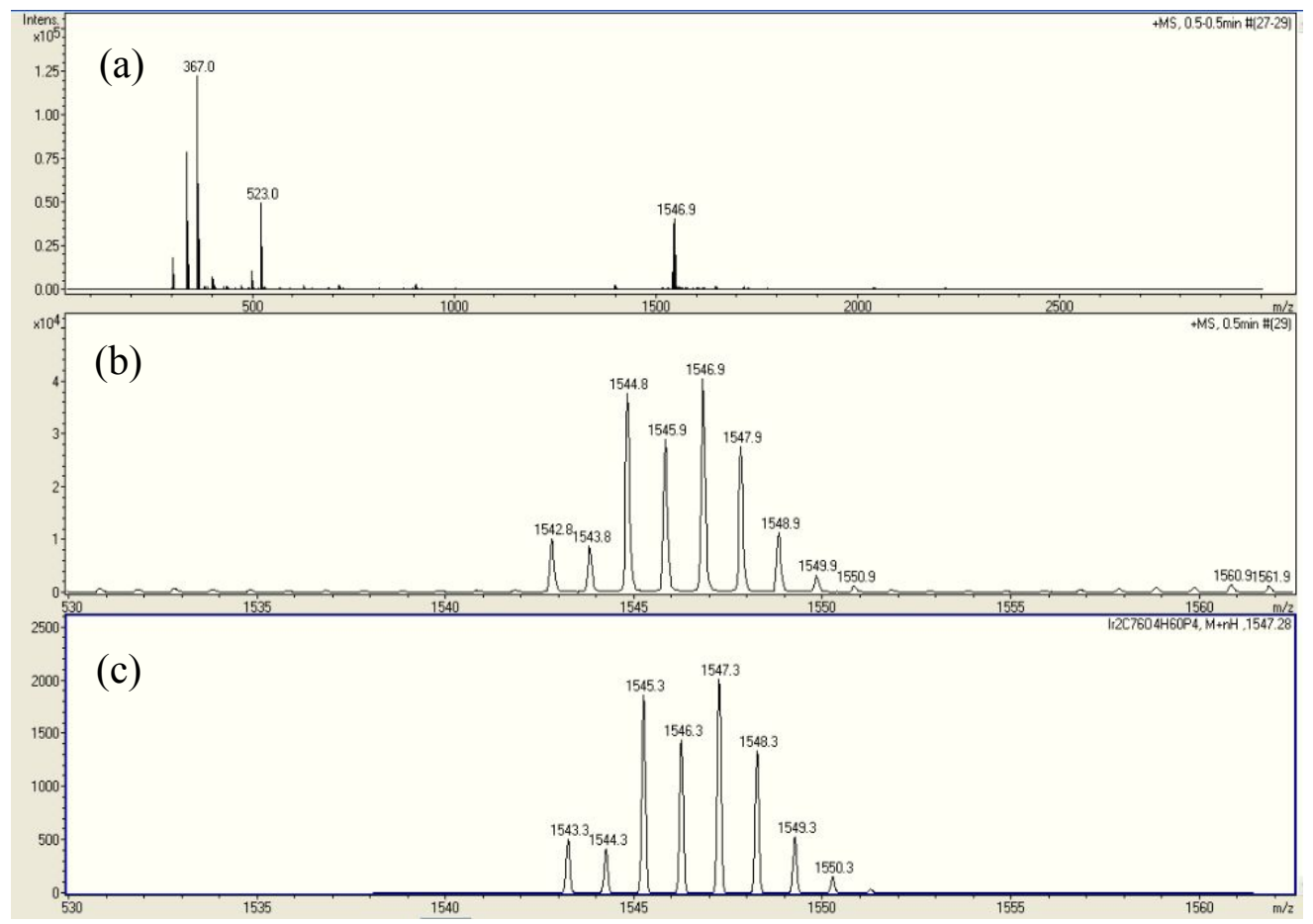

Figure S16. ESI-MS spectra of intermediate B. (a), (b); measured. (c); simulated. 


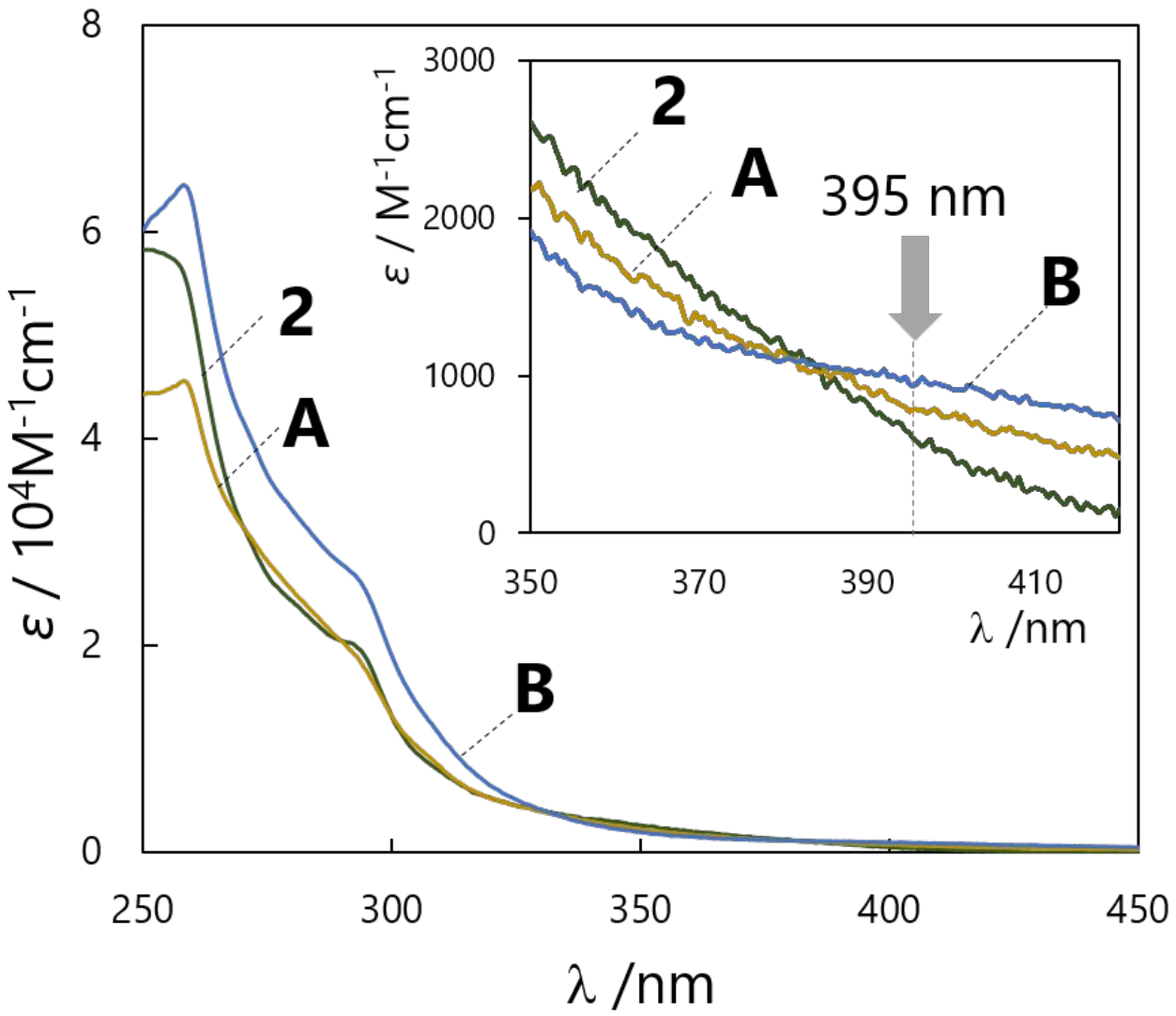

Figure S17. UV-vis absorption spectra of $\mathbf{2}, \mathbf{A}$ (and $\mathbf{A}^{\prime}$ ), and $\mathbf{B}$ (and $\mathbf{B}^{\prime}$ ) measured in $\mathrm{CH}_{2} \mathrm{Cl}_{2}$ at room temperature. 
2: after $\underline{10}$ min under $D_{2}$ (1 atm), dark condition (OFF)
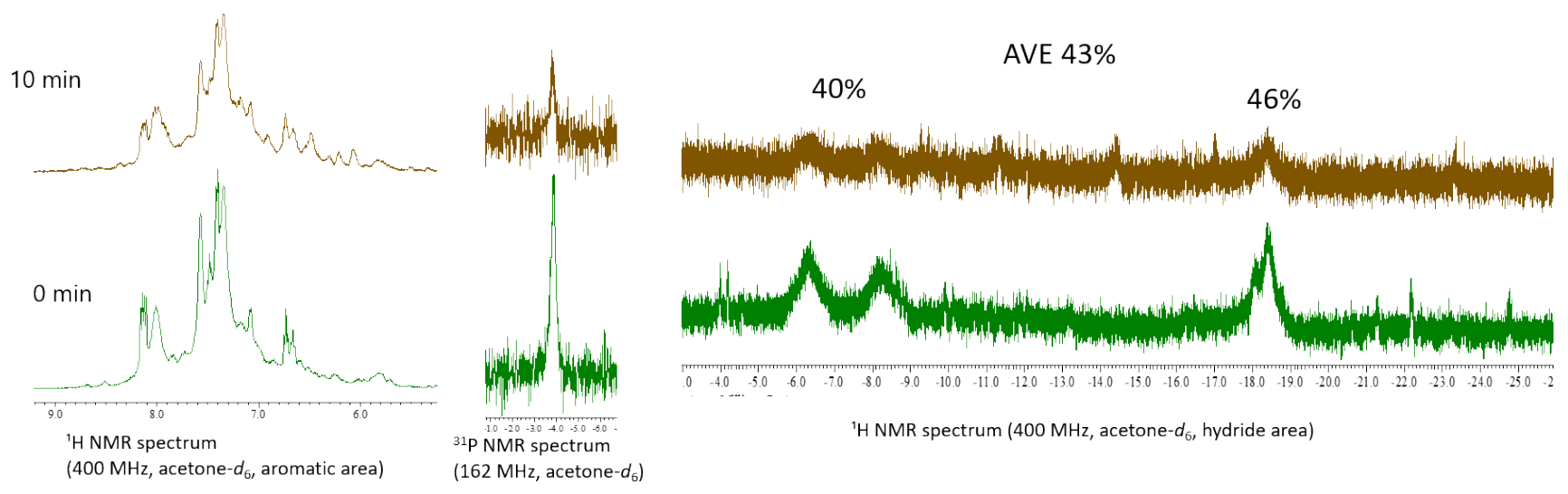

2: after 1 min under $D_{2}$ (1 atm), irradiated condition (395 nm, ON)
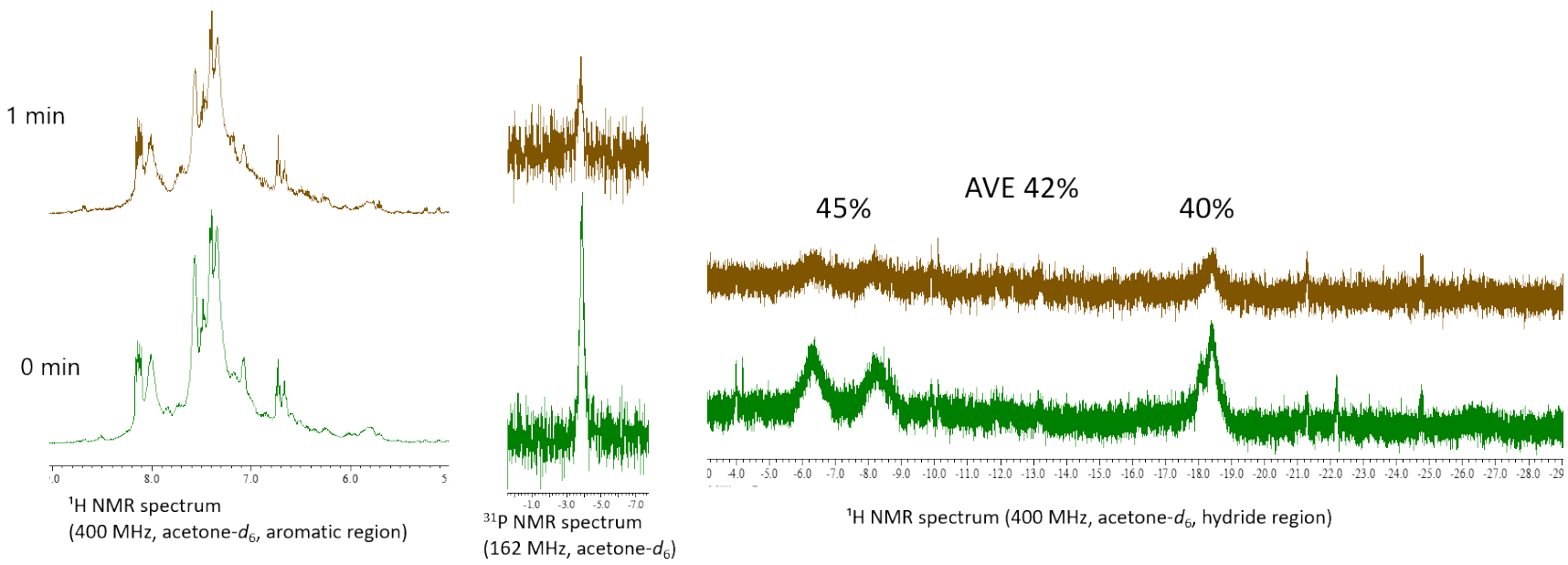

Figure S18. ${ }^{1} \mathrm{H}$ and ${ }^{31} \mathrm{P}$ NMR spectra of $2(7 \mathrm{mM})$ after the $\mathrm{H}-\mathrm{D}$ exchange reaction under irradiation $(395 \mathrm{~nm}, 1$ minute irradiation) and dark condition (10 minutes) conducted in acetone- $h_{6}$. D-solvent is only used for NMR measurements. 


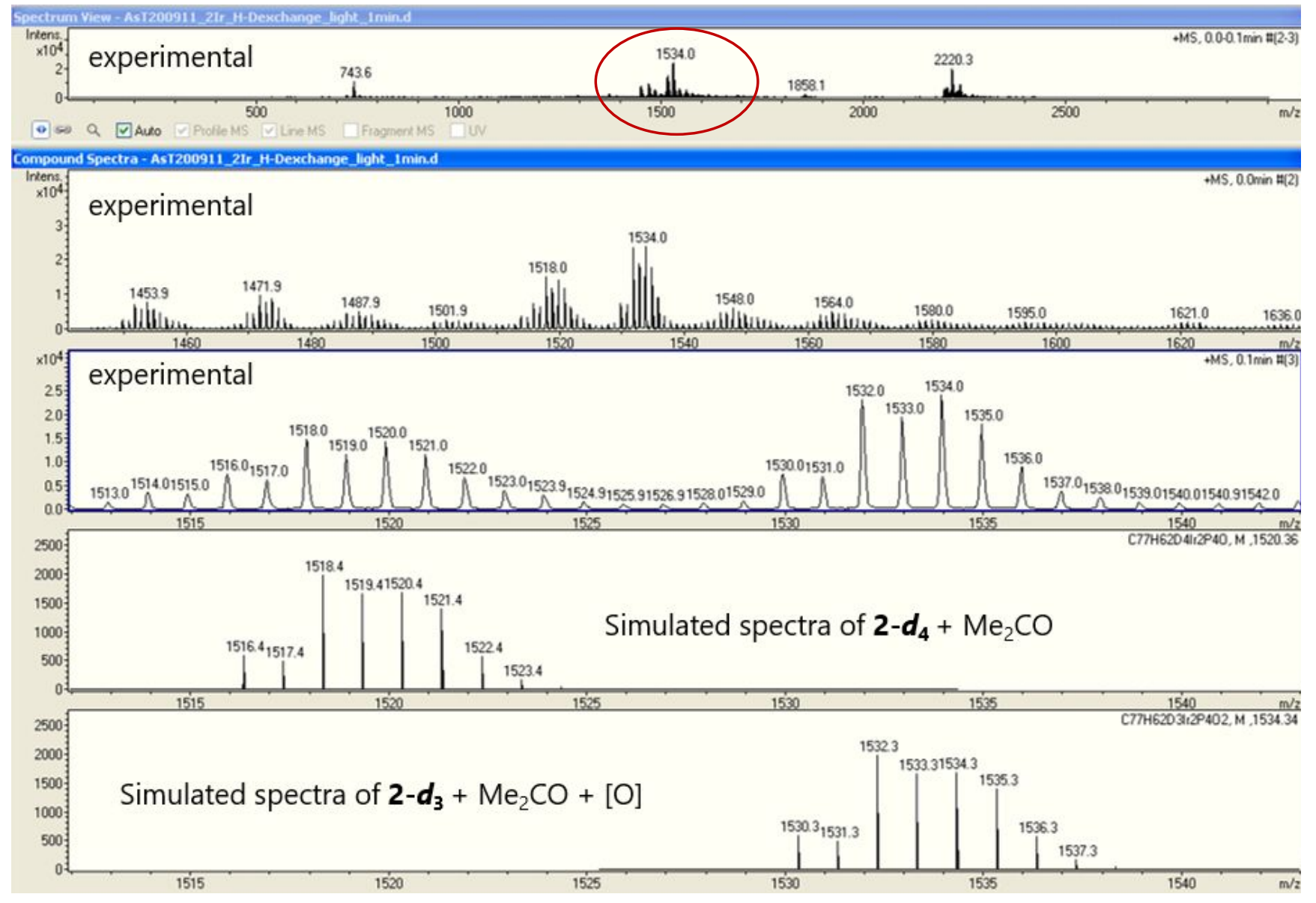

Figure S19. ESI-MS spectra of 2 after H-D exchange reaction under irradiated (395 nm) condition. 


\section{DFT calculation}

DFT calculations were performed using the Gaussian-16 Revision A.03 quantum chemistry program package $^{i}$ at the B3LYP/LanL2DZ levelii,iii. We used the LanL2DZ pseudo-potential for $\operatorname{Ir}, 6-31 \mathrm{G}(\mathrm{d})^{\mathrm{iv}}$ split-valence basis set for $\mathrm{P}$, and $3-21 \mathrm{G}^{\mathrm{v}}$ for $\mathrm{C}$ and $\mathrm{H}$. The orbital energies were determined by using minimized singlet geometries to approximate the ground state.
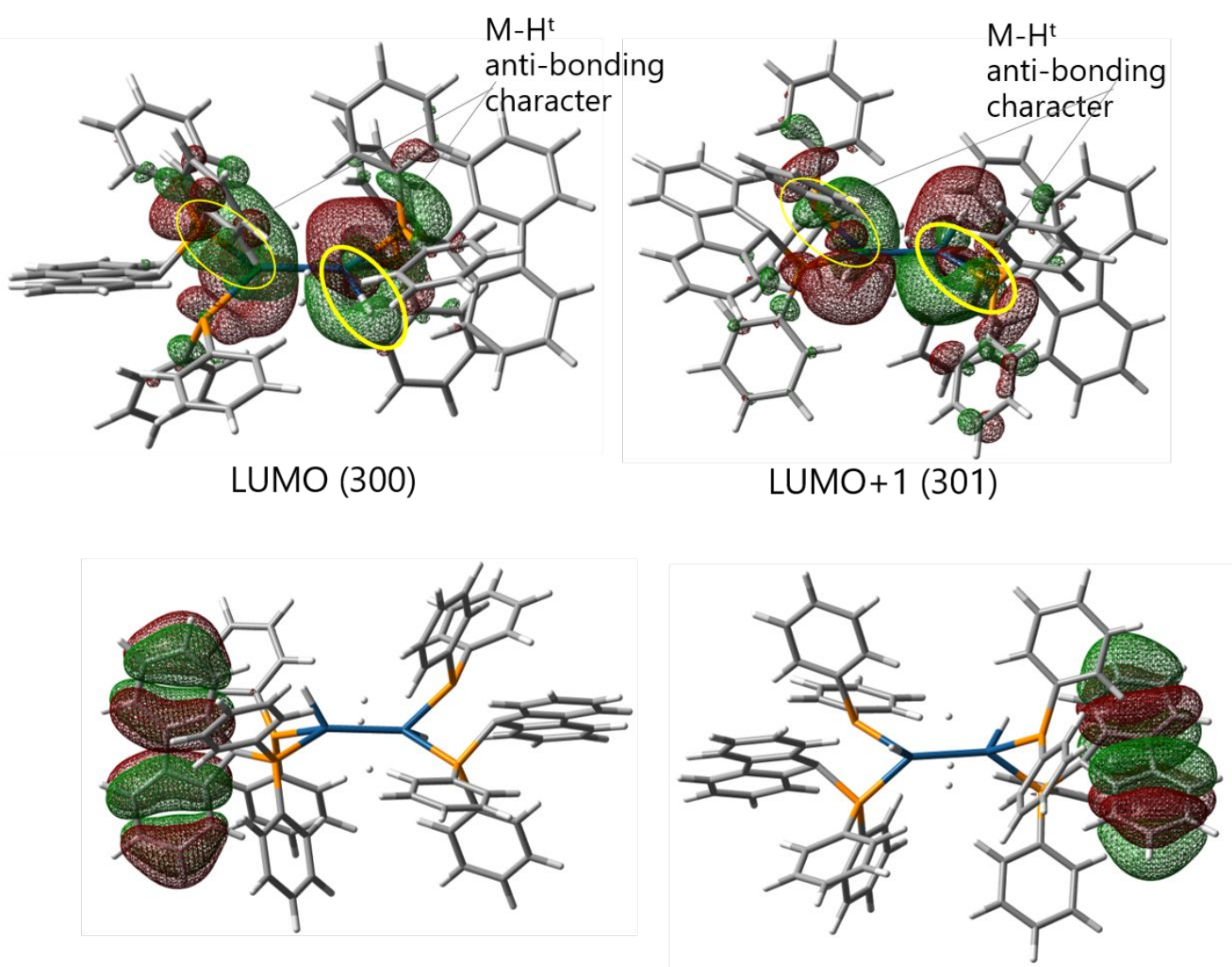

HOMO (299)

HOMO-1 (298)

Figure S20. Frontier molecular orbitals of 2. 


\section{TDDFT calculation}

TDDFT calculations were performed using the optimized structure by the Gaussian-16 Revision A.03 quantum chemistry program package ${ }^{\text {vi }}$ at the B3LYP/LanL2DZ level vii,viii.

\section{TDDFT}

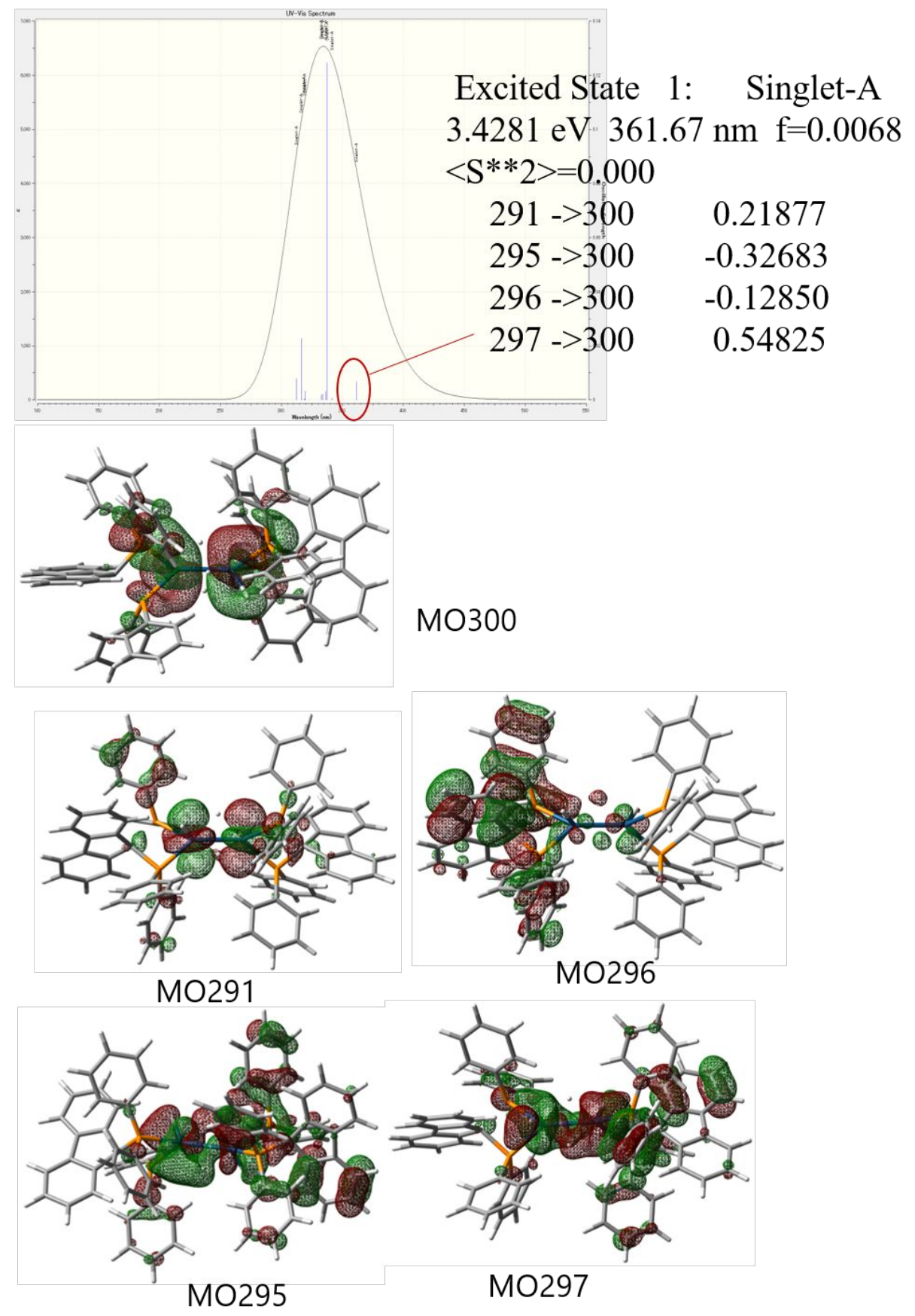

Figure S21. Preliminary TDDFT result for $\mathbf{2}$. 


\section{$X$-ray Structural Determinations.}

The diffraction data of B-OMe were collected on a Rigaku XtaLAB P100 diffractometer with graphite monochromated $\operatorname{MoK} \alpha(\lambda=0.71073 \AA)$. The data were collected at a temperature of $-100 \pm 1{ }^{\circ} \mathrm{C}$ to a maximum $2 \theta$ value of $55.1^{\circ}$. The crystal-to-detector distance was $45.00 \mathrm{~mm}$. Readout was performed in the $0.172 \mathrm{~mm}$ pixel mode. Data were collected and processed using CrystalClear (Rigaku). ${ }^{1}$ An empirical absorption correction was applied The data were corrected for Lorentz and polarization effects.

The crystal structures were solved by direct method (SHELXS-972 or SHELXT ${ }^{3}$ ) and expanded using Fourier techniques, which are subsequently completed by Fourier recycling using the SHELXL 2014 program. ${ }^{3}$ Non-hydrogen atoms were refined by anisotropic displacement parameters. Crystallographic data, data collection and refinement parameters for B-OMe are listed in Table S1 and bond lengths and angles are listed in Table S2.

(1) CrystalClear: Data Collection and Processing Software, Rigaku Corporation (1998-2015). Tokyo 1968666, Japan.

(2) SHELXS Version 2013/1: Sheldrick, G. M. (2008). Acta Cryst. A64, 112-122.

(3) SHELXT Version 2014/5: Sheldrick, G. M. (2014). Acta Cryst. A70, C1437.

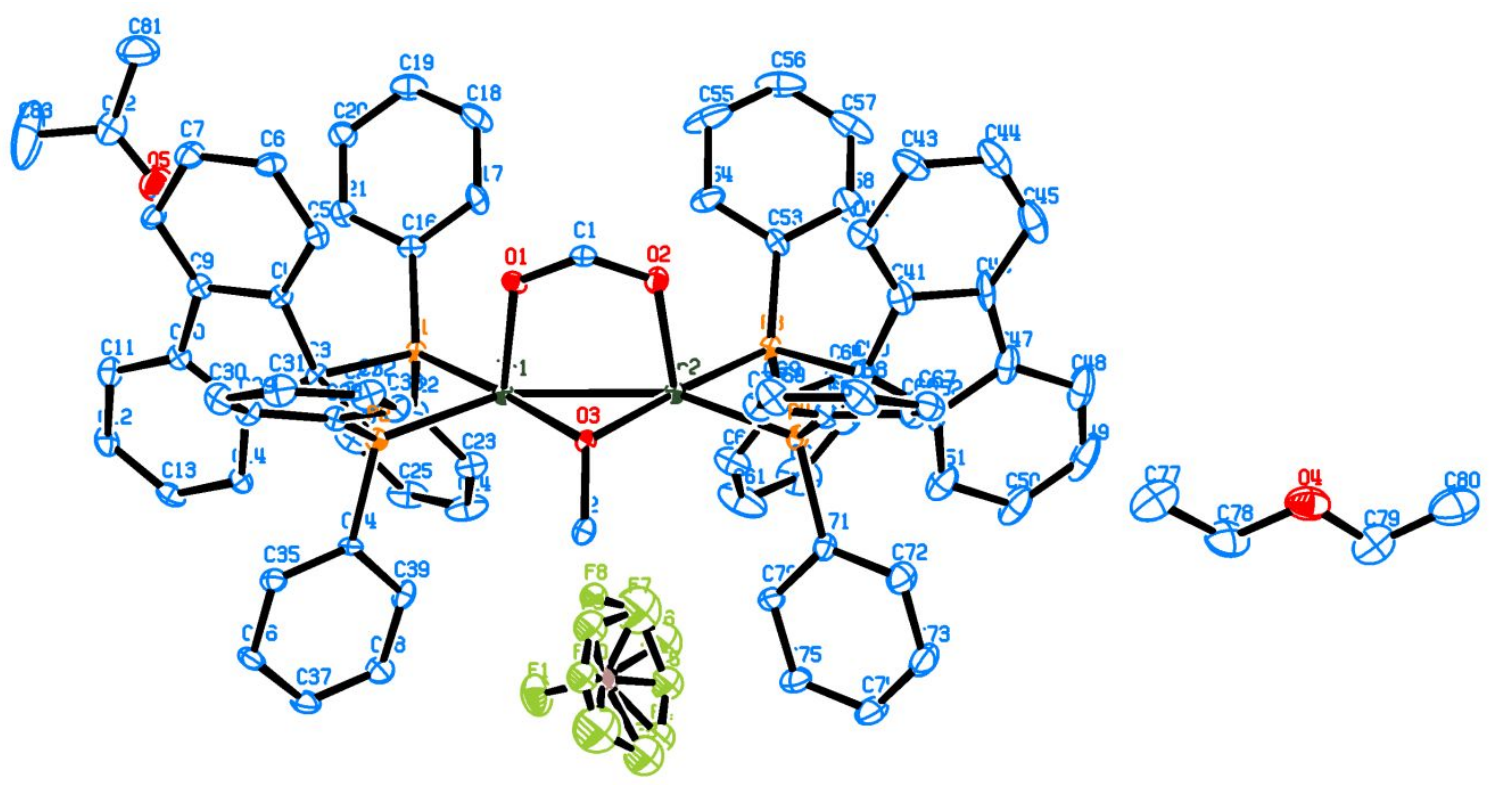

Figure S22. ORTEP diagram of B-OMe. 
Table S1. Crystal data and structure refinement for B-OMe.

\begin{tabular}{|l|l|}
\hline Complex & B-OMe. \\
\hline Empirical formula & C83 H75 B F4 Ir2 O5 P4 \\
\hline Formula weight & 1747.52 \\
\hline Temperature & $100(2) \mathrm{K}$ \\
\hline Wavelength & $0.71075 \AA$ \\
\hline Crystal system & Monoclinic \\
\hline Space group & $P 21 / \mathrm{n}$ \\
\hline Unit cell dimensions & $\mathrm{a}=11.5096(8) \AA$ \\
& $\mathrm{b}=25.4620(16) \AA$ \\
$\mathrm{c}=24.8314(17) \AA$ \\
\hline & $\alpha=90^{\circ}$ \\
\hline & $\beta=92.1449(15)^{\circ}$ \\
\hline Volume & $\gamma=90^{\circ}$ \\
\hline Z & $7271.9(8) \AA^{3}$ \\
\hline Density (calculated) & 4 \\
\hline Absorption coefficient & $1.596 \mathrm{Mg} / \mathrm{m}$ \\
\hline F(000) & $3.807 \mathrm{~mm}{ }^{-1}$ \\
\hline Crystal size & 3472 \\
\hline Theta range for data collection & $0.190 \times 0.150 \times 0.130 \mathrm{~mm}$ \\
\hline Index ranges & 3.076 to $27.519^{\circ}$. \\
\hline Reflections collected & $-14<=\mathrm{h}<=13,-32<=\mathrm{k}<=30,-32<=1<=31$ \\
\hline Independent reflections & 58227 \\
\hline Completeness to theta $=27.50^{\circ}$ & $15397[\mathrm{R}($ int $)=0.2039]$ \\
\hline Refinement method & $98.5 \%$ \\
\hline Data / restraints / parameters & Full-matrix least-squares on $\mathrm{F}^{2}$ \\
\hline Goodness-of-fit on $\mathrm{F}^{2}$ & $15397 / 0 / 910$ \\
\hline Final R indices $[\mathrm{I}>2$ sigma(I) $]$ & 1.023 \\
\hline R indices (all data) & $R 1=0.0624, w R 2=0.1766$ \\
\hline Largest diff. peak and hole & $R 1=0.0673, w R 2=0.1801$ \\
\hline & 4.184 and -5.879 e. $\AA^{-}$ \\
\hline & \\
\hline
\end{tabular}


Table S2. Atomic coordinates ( $\left.\times 10^{4}\right)$ and equivalent isotropic displacement parameters $\left(\AA^{2} \times 10^{3}\right)$ for B-OMe. U(eq) is defined as one third of the trace of the orthogonalized $\mathrm{U}^{\mathrm{ij}}$ tensor.

\begin{tabular}{|c|c|c|c|c|}
\hline & $\mathrm{x}$ & $\mathrm{y}$ & $\mathrm{z}$ & $\mathrm{U}(\mathrm{eq})$ \\
\hline $\operatorname{Ir}(1)$ & $5180(1)$ & $3974(1)$ & $6625(1)$ & $16(1)$ \\
\hline $\operatorname{Ir}(2)$ & $4297(1)$ & $4674(1)$ & $7368(1)$ & $17(1)$ \\
\hline $\mathrm{P}(1)$ & $6910(1)$ & $3893(1)$ & $6252(1)$ & $17(1)$ \\
\hline $\mathrm{P}(2)$ & $5131(1)$ & $3205(1)$ & $6168(1)$ & $18(1)$ \\
\hline $\mathrm{P}(3)$ & $5034(1)$ & $5375(1)$ & $7804(1)$ & $21(1)$ \\
\hline $\mathrm{P}(4)$ & 2973(1) & $4905(1)$ & $7973(1)$ & $21(1)$ \\
\hline $\mathrm{O}(1)$ & $4202(3)$ & $4475(2)$ & $6040(2)$ & $21(1)$ \\
\hline$C(1)$ & $3603(5)$ & 4851(3) & $6181(3)$ & $21(1)$ \\
\hline $\mathrm{O}(2)$ & $3494(4)$ & $5051(2)$ & $6640(2)$ & $25(1)$ \\
\hline $\mathrm{O}(3)$ & $3572(3)$ & $4008(2)$ & $6997(2)$ & $16(1)$ \\
\hline$C(2)$ & $3239(5)$ & $3566(3)$ & $7322(2)$ & $25(1)$ \\
\hline$C(3)$ & $6501(5)$ & $3320(2)$ & $5782(2)$ & $18(1)$ \\
\hline$C(4)$ & $6243(5)$ & $3523(2)$ & $5210(2)$ & $18(1)$ \\
\hline$C(5)$ & $5508(5)$ & $3923(2)$ & $5027(3)$ & $22(1)$ \\
\hline$C(6)$ & $5492(6)$ & $4050(3)$ & $4486(3)$ & $28(2)$ \\
\hline$C(7)$ & $6172(6)$ & $3788(3)$ & $4129(3)$ & $32(2)$ \\
\hline$C(8)$ & $6904(6)$ & $3384(3)$ & $4305(3)$ & $28(1)$ \\
\hline$C(9)$ & $6942(5)$ & $3255(3)$ & $4849(3)$ & $22(1)$ \\
\hline$C(10)$ & $7641(5)$ & 2861(3) & $5148(3)$ & $22(1)$ \\
\hline $\mathrm{C}(11)$ & $8467(6)$ & $2520(3)$ & $4968(3)$ & $30(2)$ \\
\hline$C(12)$ & $9029(6)$ & $2187(3)$ & $5336(3)$ & $30(2)$ \\
\hline$C(13)$ & $8734(6)$ & $2196(3)$ & $5877(3)$ & $29(2)$ \\
\hline$C(14)$ & $7919(5)$ & $2543(2)$ & $6064(3)$ & $23(1)$ \\
\hline$C(15)$ & $7369(5)$ & $2884(2)$ & $5697(3)$ & $21(1)$ \\
\hline$C(16)$ & $7472(5)$ & $4414(3)$ & $5835(3)$ & $23(1)$ \\
\hline$C(17)$ & $7031(6)$ & $4914(3)$ & $5897(3)$ & $31(2)$ \\
\hline$C(18)$ & $7412(7)$ & $5327(3)$ & $5576(4)$ & $41(2)$ \\
\hline$C(19)$ & $8228(7)$ & $5232(3)$ & $5194(3)$ & $40(2)$ \\
\hline$C(20)$ & $8654(6)$ & $4726(3)$ & $5123(3)$ & $32(2)$ \\
\hline $\mathrm{C}(21)$ & $8289(5)$ & $4323(3)$ & $5436(3)$ & $27(1)$ \\
\hline
\end{tabular}




\begin{tabular}{|c|c|c|c|c|}
\hline$C(22)$ & $8138(5)$ & $3674(2)$ & $6690(3)$ & $23(1)$ \\
\hline$C(23)$ & $8033(5)$ & $3651(3)$ & $7247(3)$ & $30(2)$ \\
\hline $\mathrm{C}(24)$ & $8980(7)$ & $3471(4)$ & $7574(3)$ & $49(2)$ \\
\hline$C(25)$ & $9995(6)$ & $3317(3)$ & $7351(3)$ & $43(2)$ \\
\hline$C(26)$ & $10115(6)$ & $3342(3)$ & $6797(4)$ & $37(2)$ \\
\hline$C(27)$ & $9198(5)$ & $3523(3)$ & $6463(3)$ & $29(2)$ \\
\hline$C(28)$ & $3929(5)$ & $3071(2)$ & $5693(2)$ & $20(1)$ \\
\hline$C(29)$ & $4023(5)$ & 2742(3) & $5246(2)$ & $23(1)$ \\
\hline $\mathrm{C}(30)$ & $3047(6)$ & $2640(3)$ & 4914(3) & $31(2)$ \\
\hline$C(31)$ & $1986(6)$ & 2871(3) & $5018(3)$ & $34(2)$ \\
\hline $\mathrm{C}(32)$ & $1897(6)$ & $3206(3)$ & $5450(3)$ & $31(2)$ \\
\hline$C(33)$ & $2859(5)$ & $3315(3)$ & $5790(3)$ & $25(1)$ \\
\hline$C(34)$ & $5390(5)$ & $2605(2)$ & $6548(2)$ & $18(1)$ \\
\hline$C(35)$ & $5329(5)$ & 2116(3) & 6293(3) & $25(1)$ \\
\hline$C(36)$ & $5649(6)$ & 1662(3) & $6576(3)$ & $30(2)$ \\
\hline$C(37)$ & $6029(7)$ & 1693(3) & $7112(3)$ & $34(2)$ \\
\hline$C(38)$ & $6054(6)$ & 2165(3) & 7364(3) & $31(2)$ \\
\hline C(39) & $5738(5)$ & 2617(3) & $7097(2)$ & $26(1)$ \\
\hline $\mathrm{C}(40)$ & $3664(5)$ & $5556(3)$ & $8172(3)$ & $26(1)$ \\
\hline $\mathrm{C}(41)$ & $3040(6)$ & 6021(3) & 7910(3) & $33(2)$ \\
\hline$C(42)$ & $2719(6)$ & 6106(3) & $7369(3)$ & $31(2)$ \\
\hline $\mathrm{C}(43)$ & $2231(6)$ & $6588(3)$ & $7227(4)$ & $43(2)$ \\
\hline $\mathrm{C}(44)$ & $2067(7)$ & 6975(3) & $7602(5)$ & $52(2)$ \\
\hline $\mathrm{C}(45)$ & $2365(7)$ & 6899(3) & $8139(5)$ & $53(3)$ \\
\hline $\mathrm{C}(46)$ & $2865(6)$ & $6419(3)$ & $8297(3)$ & $39(2)$ \\
\hline $\mathrm{C}(47)$ & $3294(6)$ & $6219(3)$ & $8829(3)$ & $40(2)$ \\
\hline $\mathrm{C}(48)$ & $3289(8)$ & 6449(4) & $9337(4)$ & $57(3)$ \\
\hline $\mathrm{C}(49)$ & $3725(10)$ & $6181(5)$ & 9766(4) & $72(4)$ \\
\hline$C(50)$ & $4207(8)$ & $5678(4)$ & $9712(3)$ & $53(3)$ \\
\hline $\mathrm{C}(51)$ & $4229(7)$ & $5451(4)$ & 9204(3) & $45(2)$ \\
\hline$C(52)$ & $3762(6)$ & $5715(3)$ & $8764(3)$ & $33(2)$ \\
\hline$C(53)$ & $5508(5)$ & $5953(2)$ & $7445(3)$ & $23(1)$ \\
\hline$C(54)$ & $5963(8)$ & $5880(4)$ & 6952(3) & $41(2)$ \\
\hline$C(55)$ & $6399(10)$ & $6310(4)$ & $6657(3)$ & $69(4)$ \\
\hline$C(56)$ & $6325(8)$ & 6808(4) & $6874(4)$ & $57(3)$ \\
\hline C(57) & $5865(7)$ & $6888(3)$ & $7375(5)$ & $53(3)$ \\
\hline
\end{tabular}




\begin{tabular}{|c|c|c|c|c|}
\hline$C(58)$ & $5455(6)$ & $6461(3)$ & $7654(4)$ & $41(2)$ \\
\hline$C(59)$ & $6202(6)$ & $5273(3)$ & $8314(3)$ & $29(2)$ \\
\hline$C(60)$ & $6644(7)$ & 4781(3) & $8421(3)$ & $41(2)$ \\
\hline $\mathrm{C}(61)$ & $7503(10)$ & 4699(4) & $8820(5)$ & $73(4)$ \\
\hline$C(62)$ & 7941(9) & 5121(4) & 9111(4) & $66(3)$ \\
\hline$C(63)$ & $7508(7)$ & $5610(4)$ & $9016(3)$ & $44(2)$ \\
\hline$C(64)$ & $6665(6)$ & $5697(3)$ & $8614(3)$ & $37(2)$ \\
\hline$C(65)$ & $1478(5)$ & $5004(3)$ & $7737(3)$ & $23(1)$ \\
\hline$C(66)$ & $673(6)$ & $5312(3)$ & $8014(3)$ & $30(2)$ \\
\hline$C(67)$ & $-502(6)$ & $5310(3)$ & $7838(3)$ & $33(2)$ \\
\hline $\mathrm{C}(68)$ & $-861(6)$ & $5025(3)$ & $7385(3)$ & $39(2)$ \\
\hline$C(69)$ & $-69(6)$ & $4732(3)$ & $7106(4)$ & $36(2)$ \\
\hline$C(70)$ & 1101(6) & $4720(3)$ & $7285(3)$ & $28(2)$ \\
\hline $\mathrm{C}(71)$ & $2863(6)$ & $4509(3)$ & $8584(3)$ & $26(1)$ \\
\hline $\mathrm{C}(72)$ & $2075(7)$ & $4642(3)$ & $8968(3)$ & $39(2)$ \\
\hline$C(73)$ & $2012(7)$ & $4357(4)$ & $9440(3)$ & $46(2)$ \\
\hline$C(74)$ & $2763(8)$ & $3944(3)$ & $9548(3)$ & $43(2)$ \\
\hline$C(75)$ & $3553(7)$ & $3806(3)$ & $9166(3)$ & $40(2)$ \\
\hline$C(76)$ & $3612(6)$ & 4084(3) & $8682(3)$ & $29(1)$ \\
\hline$C(77)$ & $665(9)$ & $5987(5)$ & $9669(4)$ & $70(3)$ \\
\hline $\mathrm{C}(78)$ & 787(9) & $6152(4)$ & $10263(4)$ & $65(3)$ \\
\hline $\mathrm{O}(4)$ & $22(5)$ & 6593(3) & $10360(3)$ & $59(2)$ \\
\hline$C(79)$ & $42(10)$ & $6709(5)$ & 10911(4) & $68(3)$ \\
\hline$C(80)$ & $-683(10)$ & $7160(5)$ & 10998(5) & $76(3)$ \\
\hline $\mathrm{C}(81)$ & $9440(13)$ & $4255(4)$ & $3772(4)$ & $79(4)$ \\
\hline$C(82)$ & $9978(6)$ & $3787(4)$ & $4042(3)$ & $41(2)$ \\
\hline$C(83)$ & $10557(14)$ & $3406(8)$ & $3707(5)$ & $134(8)$ \\
\hline $\mathrm{O}(5)$ & $9870(5)$ & $3711(3)$ & $4524(2)$ & $54(2)$ \\
\hline $\mathrm{B}(1)$ & $6242(9)$ & $3106(4)$ & $8642(4)$ & $43(2)$ \\
\hline $\mathrm{F}(1)$ & $7129(5)$ & $2769(2)$ & $8519(2)$ & $61(2)$ \\
\hline$F(2)$ & $5250(20)$ & $2788(11)$ & $8649(11)$ & $101(7)$ \\
\hline$F(3)$ & $5613(19)$ & 2883(9) & 9101(9) & $71(6)$ \\
\hline $\mathrm{F}(4)$ & $5983(14)$ & $3061(7)$ & $9177(6)$ & $41(3)$ \\
\hline$F(5)$ & $6446(15)$ & $3379(8)$ & $9056(7)$ & $50(4)$ \\
\hline$F(6)$ & $6810(20)$ & $3576(9)$ & $8906(10)$ & $79(6)$ \\
\hline $\mathrm{F}(7)$ & $6430(20)$ & $3606(11)$ & $8549(12)$ & $104(7)$ \\
\hline
\end{tabular}




$\begin{array}{lllll}\mathrm{F}(8) & 6088(12) & 3455(6) & 8185(5) & 33(3) \\ \mathrm{F}(9) & 5632(16) & 3269(7) & 8222(6) & 49(4) \\ \mathrm{F}(10) & 5205(13) & 2975(6) & 8327(6) & 42(3)\end{array}$


Table S3. Bond lengths $[\AA]$ and angles $\quad \mathrm{C}(5)-\mathrm{C}(6)$

$1.380(9)$ $\left.{ }^{\circ}\right]$ for B-OMe.

\begin{tabular}{|c|c|c|c|}
\hline & & $\mathrm{C}(5)-\mathrm{H}(5)$ & 0.9500 \\
\hline $\operatorname{Ir}(1)-\mathrm{O}(3)$ & $2.101(4)$ & $C(6)-C(7)$ & $1.378(10)$ \\
\hline $\operatorname{Ir}(1)-\mathrm{O}(1)$ & $2.209(4)$ & $\mathrm{C}(6)-\mathrm{H}(6)$ & 0.9500 \\
\hline $\operatorname{Ir}(1)-\mathrm{P}(1)$ & $2.2370(16)$ & $\mathrm{C}(7)-\mathrm{C}(8)$ & $1.390(9)$ \\
\hline $\operatorname{Ir}(1)-\mathrm{P}(2)$ & $2.2626(16)$ & $\mathrm{C}(7)-\mathrm{H}(7)$ & 0.9500 \\
\hline $\operatorname{Ir}(1)-\operatorname{Ir}(2)$ & $2.7846(3)$ & $\mathrm{C}(8)-\mathrm{C}(9)$ & $1.389(9)$ \\
\hline $\operatorname{Ir}(2)-\mathrm{O}(3)$ & $2.090(4)$ & $\mathrm{C}(8)-\mathrm{H}(8)$ & 0.9500 \\
\hline $\operatorname{Ir}(2)-\mathrm{O}(2)$ & $2.216(4)$ & $\mathrm{C}(9)-\mathrm{C}(10)$ & $1.470(9)$ \\
\hline $\operatorname{Ir}(2)-P(3)$ & $2.2376(16)$ & $C(10)-C(11)$ & $1.375(9)$ \\
\hline $\operatorname{Ir}(2)-P(4)$ & $2.2569(16)$ & $C(10)-C(15)$ & $1.410(9)$ \\
\hline $\mathrm{P}(1)-\mathrm{C}(16)$ & $1.818(7)$ & $C(11)-C(12)$ & $1.386(9)$ \\
\hline $\mathrm{P}(1)-\mathrm{C}(22)$ & $1.837(6)$ & $\mathrm{C}(11)-\mathrm{H}(11)$ & 0.9500 \\
\hline $\mathrm{P}(1)-\mathrm{C}(3)$ & $1.916(6)$ & $C(12)-C(13)$ & $1.398(10)$ \\
\hline $\mathrm{P}(1)-\mathrm{P}(2)$ & $2.696(2)$ & $\mathrm{C}(12)-\mathrm{H}(12)$ & 0.9500 \\
\hline $\mathrm{P}(2)-\mathrm{C}(34)$ & $1.814(6)$ & $C(13)-C(14)$ & $1.383(9)$ \\
\hline $\mathrm{P}(2)-\mathrm{C}(28)$ & $1.817(5)$ & $\mathrm{C}(13)-\mathrm{H}(13)$ & 0.9500 \\
\hline $\mathrm{P}(2)-\mathrm{C}(3)$ & $1.899(6)$ & $C(14)-C(15)$ & $1.392(8)$ \\
\hline $\mathrm{P}(3)-\mathrm{C}(53)$ & $1.815(7)$ & $\mathrm{C}(14)-\mathrm{H}(14)$ & 0.9500 \\
\hline $\mathrm{P}(3)-\mathrm{C}(59)$ & $1.831(7)$ & $C(16)-C(17)$ & $1.380(10)$ \\
\hline $\mathrm{P}(3)-\mathrm{C}(40)$ & $1.908(7)$ & $C(16)-C(21)$ & $1.412(10)$ \\
\hline $\mathrm{P}(4)-\mathrm{C}(65)$ & $1.815(6)$ & $C(17)-C(18)$ & $1.399(11)$ \\
\hline $\mathrm{P}(4)-\mathrm{C}(71)$ & $1.830(7)$ & $\mathrm{C}(17)-\mathrm{H}(17)$ & 0.9500 \\
\hline $\mathrm{P}(4)-\mathrm{C}(40)$ & $1.896(7)$ & $C(18)-C(19)$ & $1.382(12)$ \\
\hline $\mathrm{O}(1)-\mathrm{C}(1)$ & $1.237(8)$ & $\mathrm{C}(18)-\mathrm{H}(18)$ & 0.9500 \\
\hline $\mathrm{C}(1)-\mathrm{O}(2)$ & $1.259(8)$ & $C(19)-C(20)$ & $1.392(11)$ \\
\hline $\mathrm{C}(1)-\mathrm{H}(4)$ & $0.86(7)$ & $\mathrm{C}(19)-\mathrm{H}(19)$ & 0.9500 \\
\hline $\mathrm{O}(3)-\mathrm{C}(2)$ & $1.445(8)$ & $C(20)-C(21)$ & $1.364(10)$ \\
\hline $\mathrm{C}(2)-\mathrm{H}(2)$ & 0.9800 & $\mathrm{C}(20)-\mathrm{H}(20)$ & 0.9500 \\
\hline $\mathrm{C}(2)-\mathrm{H}(2 \mathrm{~A})$ & 0.9800 & $\mathrm{C}(21)-\mathrm{H}(21)$ & 0.9500 \\
\hline $\mathrm{C}(2)-\mathrm{H}(2 \mathrm{~B})$ & 0.9800 & $C(22)-C(23)$ & $1.393(10)$ \\
\hline$C(3)-C(15)$ & $1.514(8)$ & $C(22)-C(27)$ & $1.415(10)$ \\
\hline $\mathrm{C}(3)-\mathrm{C}(4)$ & $1.531(8)$ & $C(23)-C(24)$ & $1.412(9)$ \\
\hline$C(4)-C(5)$ & $1.390(8)$ & $\mathrm{C}(23)-\mathrm{H}(23)$ & 0.9500 \\
\hline$C(4)-C(9)$ & $1.404(9)$ & $C(24)-C(25)$ & $1.369(13)$ \\
\hline
\end{tabular}




\begin{tabular}{|c|c|c|c|}
\hline $\mathrm{C}(24)-\mathrm{H}(24)$ & 0.9500 & $C(44)-C(45)$ & $1.380(15)$ \\
\hline$C(25)-C(26)$ & $1.390(12)$ & $\mathrm{C}(44)-\mathrm{H}(44)$ & 0.9500 \\
\hline $\mathrm{C}(25)-\mathrm{H}(25)$ & 0.9500 & $C(45)-C(46)$ & $1.400(13)$ \\
\hline$C(26)-C(27)$ & $1.395(9)$ & $\mathrm{C}(45)-\mathrm{H}(45)$ & 0.9500 \\
\hline $\mathrm{C}(26)-\mathrm{H}(26)$ & 0.9500 & $C(46)-C(47)$ & $1.483(12)$ \\
\hline $\mathrm{C}(27)-\mathrm{H}(27)$ & 0.9500 & $\mathrm{C}(47)-\mathrm{C}(48)$ & $1.391(11)$ \\
\hline $\mathrm{C}(28)-\mathrm{C}(29)$ & $1.400(9)$ & $C(47)-C(52)$ & $1.403(12)$ \\
\hline $\mathrm{C}(28)-\mathrm{C}(33)$ & $1.407(9)$ & $\mathrm{C}(48)-\mathrm{C}(49)$ & $1.346(16)$ \\
\hline C(29)-C(30) & $1.393(8)$ & $\mathrm{C}(48)-\mathrm{H}(48)$ & 0.9500 \\
\hline C(29)-H(29) & 0.9500 & $C(49)-C(50)$ & $1.404(17)$ \\
\hline $\mathrm{C}(30)-\mathrm{C}(31)$ & $1.387(11)$ & C(49)-H(49) & 0.9500 \\
\hline $\mathrm{C}(30)-\mathrm{H}(30)$ & 0.9500 & $C(50)-C(51)$ & $1.388(11)$ \\
\hline C(31)-C(32) & $1.378(11)$ & $\mathrm{C}(50)-\mathrm{H}(50)$ & 0.9500 \\
\hline $\mathrm{C}(31)-\mathrm{H}(31)$ & 0.9500 & $\mathrm{C}(51)-\mathrm{C}(52)$ & $1.373(12)$ \\
\hline C(32)-C(33) & $1.395(8)$ & $\mathrm{C}(51)-\mathrm{H}(51)$ & 0.9500 \\
\hline $\mathrm{C}(32)-\mathrm{H}(32)$ & 0.9500 & $C(53)-C(54)$ & $1.360(11)$ \\
\hline C(33)-H(33) & 0.9500 & $\mathrm{C}(53)-\mathrm{C}(58)$ & $1.396(10)$ \\
\hline $\mathrm{C}(34)-\mathrm{C}(35)$ & $1.398(9)$ & $\mathrm{C}(54)-\mathrm{C}(55)$ & $1.419(12)$ \\
\hline C(34)-C(39) & $1.407(8)$ & $\mathrm{C}(54)-\mathrm{H}(54)$ & 0.9500 \\
\hline$C(35)-C(36)$ & $1.396(9)$ & $\mathrm{C}(55)-\mathrm{C}(56)$ & $1.382(16)$ \\
\hline $\mathrm{C}(35)-\mathrm{H}(35)$ & 0.9500 & $\mathrm{C}(55)-\mathrm{H}(84)$ & 0.9500 \\
\hline$C(36)-C(37)$ & $1.387(10)$ & $C(56)-C(57)$ & $1.385(15)$ \\
\hline $\mathrm{C}(36)-\mathrm{H}(36)$ & 0.9500 & $\mathrm{C}(56)-\mathrm{H}(56)$ & 0.9500 \\
\hline $\mathrm{C}(37)-\mathrm{C}(38)$ & $1.355(10)$ & $\mathrm{C}(57)-\mathrm{C}(58)$ & $1.381(12)$ \\
\hline $\mathrm{C}(37)-\mathrm{H}(37)$ & 0.9500 & $\mathrm{C}(57)-\mathrm{H}(57)$ & 0.9500 \\
\hline C(38)-C(39) & $1.371(10)$ & $\mathrm{C}(58)-\mathrm{H}(58)$ & 0.9500 \\
\hline $\mathrm{C}(38)-\mathrm{H}(38)$ & 0.9500 & $\mathrm{C}(59)-\mathrm{C}(60)$ & $1.375(10)$ \\
\hline C(39)-H(39) & 0.9500 & $\mathrm{C}(59)-\mathrm{C}(64)$ & $1.405(10)$ \\
\hline $\mathrm{C}(40)-\mathrm{C}(41)$ & $1.520(10)$ & $\mathrm{C}(60)-\mathrm{C}(61)$ & $1.388(10)$ \\
\hline $\mathrm{C}(40)-\mathrm{C}(52)$ & $1.525(9)$ & $\mathrm{C}(60)-\mathrm{H}(60)$ & 0.9500 \\
\hline$C(41)-C(42)$ & $1.397(11)$ & $C(61)-C(62)$ & $1.380(13)$ \\
\hline$C(41)-C(46)$ & $1.417(10)$ & $\mathrm{C}(61)-\mathrm{H}(61)$ & 0.9500 \\
\hline$C(42)-C(43)$ & $1.389(10)$ & $C(62)-C(63)$ & $1.359(14)$ \\
\hline $\mathrm{C}(42)-\mathrm{H}(42)$ & 0.9500 & $\mathrm{C}(62)-\mathrm{H}(62)$ & 0.9500 \\
\hline C(43)-C(44) & $1.374(13)$ & $\mathrm{C}(63)-\mathrm{C}(64)$ & $1.382(10)$ \\
\hline C(43)-H(43) & 0.9500 & $\mathrm{C}(63)-\mathrm{H}(63)$ & 0.9500 \\
\hline
\end{tabular}




\begin{tabular}{|c|c|c|c|}
\hline $\mathrm{C}(64)-\mathrm{H}(64)$ & 0.9500 & $\mathrm{C}(80)-\mathrm{H}(80 \mathrm{~B})$ & 0.9800 \\
\hline$C(65)-C(70)$ & $1.391(9)$ & $\mathrm{C}(81)-\mathrm{C}(82)$ & $1.491(14)$ \\
\hline$C(65)-C(66)$ & $1.411(9)$ & $\mathrm{C}(81)-\mathrm{H}(81)$ & 0.9800 \\
\hline$C(66)-C(67)$ & $1.405(10)$ & $\mathrm{C}(81)-\mathrm{H}(81 \mathrm{~A})$ & 0.9800 \\
\hline $\mathrm{C}(66)-\mathrm{H}(66)$ & 0.9500 & $\mathrm{C}(81)-\mathrm{H}(81 \mathrm{~B})$ & 0.9800 \\
\hline$C(67)-C(68)$ & $1.390(11)$ & $\mathrm{C}(82)-\mathrm{O}(5)$ & $1.223(10)$ \\
\hline $\mathrm{C}(67)-\mathrm{H}(67)$ & 0.9500 & $\mathrm{C}(82)-\mathrm{C}(83)$ & $1.456(15)$ \\
\hline $\mathrm{C}(68)-\mathrm{C}(69)$ & $1.385(11)$ & $\mathrm{C}(83)-\mathrm{H}(83)$ & 0.9800 \\
\hline $\mathrm{C}(68)-\mathrm{H}(68)$ & 0.9500 & $\mathrm{C}(83)-\mathrm{H}(83 \mathrm{~A})$ & 0.9800 \\
\hline $\mathrm{C}(69)-\mathrm{C}(70)$ & $1.402(9)$ & $\mathrm{C}(83)-\mathrm{H}(83 \mathrm{~B})$ & 0.9800 \\
\hline C(69)-H(69) & 0.9500 & $\mathrm{~B}(1)-\mathrm{F}(5)$ & $1.255(18)$ \\
\hline $\mathrm{C}(70)-\mathrm{H}(70)$ & 0.9500 & $\mathrm{~B}(1)-\mathrm{F}(9)$ & $1.303(17)$ \\
\hline$C(71)-C(72)$ & $1.383(11)$ & $\mathrm{B}(1)-\mathrm{F}(7)$ & $1.31(3)$ \\
\hline$C(71)-C(76)$ & $1.398(10)$ & $\mathrm{B}(1)-\mathrm{F}(4)$ & $1.377(18)$ \\
\hline$C(72)-C(73)$ & $1.383(11)$ & $\mathrm{B}(1)-\mathrm{F}(1)$ & $1.377(11)$ \\
\hline $\mathrm{C}(72)-\mathrm{H}(72)$ & 0.9500 & $\mathrm{~B}(1)-\mathrm{F}(2)$ & $1.40(3)$ \\
\hline$C(73)-C(74)$ & $1.382(12)$ & $\mathrm{B}(1)-\mathrm{F}(10)$ & $1.441(17)$ \\
\hline $\mathrm{C}(73)-\mathrm{H}(73)$ & 0.9500 & $\mathrm{~B}(1)-\mathrm{F}(8)$ & $1.448(16)$ \\
\hline$C(74)-C(75)$ & $1.383(12)$ & $\mathrm{B}(1)-\mathrm{F}(3)$ & $1.49(2)$ \\
\hline $\mathrm{C}(74)-\mathrm{H}(74)$ & 0.9500 & $\mathrm{~B}(1)-\mathrm{F}(6)$ & $1.50(3)$ \\
\hline$C(75)-C(76)$ & $1.399(10)$ & $\mathrm{F}(2)-\mathrm{F}(10)$ & $0.93(3)$ \\
\hline $\mathrm{C}(75)-\mathrm{H}(75)$ & 0.9500 & $F(2)-F(3)$ & $1.21(3)$ \\
\hline $\mathrm{C}(76)-\mathrm{H}(76)$ & 0.9500 & $F(2)-F(4)$ & $1.68(3)$ \\
\hline $\mathrm{C}(77)-\mathrm{C}(78)$ & $1.536(15)$ & $F(2)-F(9)$ & $1.69(3)$ \\
\hline $\mathrm{C}(77)-\mathrm{H}(77)$ & 0.9800 & $\mathrm{~F}(3)-\mathrm{F}(4)$ & $0.64(2)$ \\
\hline $\mathrm{C}(77)-\mathrm{H}(77 \mathrm{~A})$ & 0.9800 & $F(3)-F(5)$ & $1.59(3)$ \\
\hline $\mathrm{C}(77)-\mathrm{H}(77 \mathrm{~B})$ & 0.9800 & $F(4)-F(5)$ & $1.02(2)$ \\
\hline $\mathrm{C}(78)-\mathrm{O}(4)$ & $1.453(12)$ & $F(4)-F(6)$ & $1.77(3)$ \\
\hline $\mathrm{C}(78)-\mathrm{H}(78)$ & 0.9900 & $F(5)-F(6)$ & $0.76(2)$ \\
\hline $\mathrm{C}(78)-\mathrm{H}(78 \mathrm{~A})$ & 0.9900 & $F(5)-F(7)$ & $1.38(3)$ \\
\hline $\mathrm{O}(4)-\mathrm{C}(79)$ & $1.400(12)$ & $F(6)-F(7)$ & $0.98(3)$ \\
\hline$C(79)-C(80)$ & $1.440(16)$ & $F(7)-F(8)$ & $1.05(3)$ \\
\hline C(79)-H(79) & 0.9900 & $F(7)-F(9)$ & $1.48(3)$ \\
\hline $\mathrm{C}(79)-\mathrm{H}(79 \mathrm{~A})$ & 0.9900 & $\mathrm{~F}(8)-\mathrm{F}(9)$ & $0.715(18)$ \\
\hline $\mathrm{C}(80)-\mathrm{H}(80)$ & 0.9800 & $\mathrm{~F}(8)-\mathrm{F}(10)$ & $1.64(2)$ \\
\hline $\mathrm{C}(80)-\mathrm{H}(80 \mathrm{~A})$ & 0.9800 & $\mathrm{~F}(9)-\mathrm{F}(10)$ & $0.938(19)$ \\
\hline
\end{tabular}




\begin{tabular}{|c|c|c|c|}
\hline & & $\mathrm{C}(3)-\mathrm{P}(2)-\operatorname{Ir}(1)$ & $96.57(19)$ \\
\hline $\mathrm{O}(3)-\operatorname{Ir}(1)-\mathrm{O}(1)$ & $80.19(16)$ & $\mathrm{C}(34)-\mathrm{P}(2)-\mathrm{P}(1)$ & $113.35(19)$ \\
\hline $\mathrm{O}(3)-\operatorname{Ir}(1)-\mathrm{P}(1)$ & $176.65(12)$ & $\mathrm{C}(28)-\mathrm{P}(2)-\mathrm{P}(1)$ & $136.9(2)$ \\
\hline $\mathrm{O}(1)-\operatorname{Ir}(1)-\mathrm{P}(1)$ & $102.77(12)$ & $\mathrm{C}(3)-\mathrm{P}(2)-\mathrm{P}(1)$ & $45.28(18)$ \\
\hline $\mathrm{O}(3)-\operatorname{Ir}(1)-\mathrm{P}(2)$ & $104.46(12)$ & $\operatorname{Ir}(1)-\mathrm{P}(2)-\mathrm{P}(1)$ & $52.75(5)$ \\
\hline $\mathrm{O}(1)-\operatorname{Ir}(1)-\mathrm{P}(2)$ & $99.61(12)$ & $\mathrm{C}(53)-\mathrm{P}(3)-\mathrm{C}(59)$ & $103.2(3)$ \\
\hline $\mathrm{P}(1)-\operatorname{Ir}(1)-\mathrm{P}(2)$ & $73.63(6)$ & $\mathrm{C}(53)-\mathrm{P}(3)-\mathrm{C}(40)$ & 108.0(3) \\
\hline $\mathrm{O}(3)-\operatorname{Ir}(1)-\operatorname{Ir}(2)$ & $48.20(11)$ & $\mathrm{C}(59)-\mathrm{P}(3)-\mathrm{C}(40)$ & $107.5(3)$ \\
\hline $\mathrm{O}(1)-\operatorname{Ir}(1)-\operatorname{Ir}(2)$ & $82.90(11)$ & $\mathrm{C}(53)-\mathrm{P}(3)-\operatorname{Ir}(2)$ & $121.6(2)$ \\
\hline$P(1)-\operatorname{Ir}(1)-\operatorname{Ir}(2)$ & 133.33(4) & $\mathrm{C}(59)-\mathrm{P}(3)-\operatorname{Ir}(2)$ & $118.5(2)$ \\
\hline$P(2)-\operatorname{Ir}(1)-\operatorname{Ir}(2)$ & $152.05(4)$ & $\mathrm{C}(40)-\mathrm{P}(3)-\operatorname{Ir}(2)$ & $96.8(2)$ \\
\hline $\mathrm{O}(3)-\operatorname{Ir}(2)-\mathrm{O}(2)$ & $81.07(16)$ & $\mathrm{C}(65)-\mathrm{P}(4)-\mathrm{C}(71)$ & $104.5(3)$ \\
\hline $\mathrm{O}(3)-\operatorname{Ir}(2)-\mathrm{P}(3)$ & $177.09(12)$ & $\mathrm{C}(65)-\mathrm{P}(4)-\mathrm{C}(40)$ & $110.3(3)$ \\
\hline $\mathrm{O}(2)-\operatorname{Ir}(2)-\mathrm{P}(3)$ & $100.87(13)$ & $\mathrm{C}(71)-\mathrm{P}(4)-\mathrm{C}(40)$ & $107.8(3)$ \\
\hline $\mathrm{O}(3)-\operatorname{Ir}(2)-\mathrm{P}(4)$ & $103.65(12)$ & $\mathrm{C}(65)-\mathrm{P}(4)-\operatorname{Ir}(2)$ & $118.5(2)$ \\
\hline $\mathrm{O}(2)-\operatorname{Ir}(2)-\mathrm{P}(4)$ & $99.12(12)$ & $\mathrm{C}(71)-\mathrm{P}(4)-\operatorname{Ir}(2)$ & $118.5(2)$ \\
\hline $\mathrm{P}(3)-\operatorname{Ir}(2)-\mathrm{P}(4)$ & $73.95(6)$ & $\mathrm{C}(40)-\mathrm{P}(4)-\operatorname{Ir}(2)$ & $96.6(2)$ \\
\hline $\mathrm{O}(3)-\operatorname{Ir}(2)-\operatorname{Ir}(1)$ & $48.53(11)$ & $\mathrm{C}(1)-\mathrm{O}(1)-\operatorname{Ir}(1)$ & $122.3(4)$ \\
\hline $\mathrm{O}(2)-\operatorname{Ir}(2)-\operatorname{Ir}(1)$ & $83.61(12)$ & $\mathrm{O}(1)-\mathrm{C}(1)-\mathrm{O}(2)$ & $130.2(6)$ \\
\hline$P(3)-\operatorname{Ir}(2)-\operatorname{Ir}(1)$ & 133.63(4) & $\mathrm{O}(1)-\mathrm{C}(1)-\mathrm{H}(4)$ & $114(5)$ \\
\hline $\mathrm{P}(4)-\operatorname{Ir}(2)-\operatorname{Ir}(1)$ & 151.59(4) & $\mathrm{O}(2)-\mathrm{C}(1)-\mathrm{H}(4)$ & $115(5)$ \\
\hline$C(16)-P(1)-C(22)$ & 106.1(3) & $\mathrm{C}(1)-\mathrm{O}(2)-\operatorname{Ir}(2)$ & $120.6(4)$ \\
\hline $\mathrm{C}(16)-\mathrm{P}(1)-\mathrm{C}(3)$ & 107.1(3) & $\mathrm{C}(2)-\mathrm{O}(3)-\operatorname{Ir}(2)$ & $119.8(3)$ \\
\hline $\mathrm{C}(22)-\mathrm{P}(1)-\mathrm{C}(3)$ & 107.2(3) & $\mathrm{C}(2)-\mathrm{O}(3)-\operatorname{Ir}(1)$ & 118.0(3) \\
\hline$C(16)-P(1)-\operatorname{Ir}(1)$ & $120.6(2)$ & $\operatorname{Ir}(2)-\mathrm{O}(3)-\operatorname{Ir}(1)$ & $83.27(14)$ \\
\hline $\mathrm{C}(22)-\mathrm{P}(1)-\operatorname{Ir}(1)$ & $117.4(2)$ & $\mathrm{O}(3)-\mathrm{C}(2)-\mathrm{H}(2)$ & 109.5 \\
\hline$C(3)-P(1)-\operatorname{Ir}(1)$ & $96.91(18)$ & $\mathrm{O}(3)-\mathrm{C}(2)-\mathrm{H}(2 \mathrm{~A})$ & 109.5 \\
\hline $\mathrm{C}(16)-\mathrm{P}(1)-\mathrm{P}(2)$ & 135.66(19) & $\mathrm{H}(2)-\mathrm{C}(2)-\mathrm{H}(2 \mathrm{~A})$ & 109.5 \\
\hline $\mathrm{C}(22)-\mathrm{P}(1)-\mathrm{P}(2)$ & $114.5(2)$ & $\mathrm{O}(3)-\mathrm{C}(2)-\mathrm{H}(2 \mathrm{~B})$ & 109.5 \\
\hline C(3)-P(1)-P(2) & $44.76(18)$ & $\mathrm{H}(2)-\mathrm{C}(2)-\mathrm{H}(2 \mathrm{~B})$ & 109.5 \\
\hline $\operatorname{Ir}(1)-\mathrm{P}(1)-\mathrm{P}(2)$ & $53.62(5)$ & $\mathrm{H}(2 \mathrm{~A})-\mathrm{C}(2)-\mathrm{H}(2 \mathrm{~B})$ & 109.5 \\
\hline $\mathrm{C}(34)-\mathrm{P}(2)-\mathrm{C}(28)$ & $106.7(3)$ & $C(15)-C(3)-C(4)$ & $102.9(5)$ \\
\hline $\mathrm{C}(34)-\mathrm{P}(2)-\mathrm{C}(3)$ & $105.6(3)$ & $\mathrm{C}(15)-\mathrm{C}(3)-\mathrm{P}(2)$ & $121.5(4)$ \\
\hline $\mathrm{C}(28)-\mathrm{P}(2)-\mathrm{C}(3)$ & 109.1(3) & $\mathrm{C}(4)-\mathrm{C}(3)-\mathrm{P}(2)$ & $112.6(4)$ \\
\hline $\mathrm{C}(34)-\mathrm{P}(2)-\operatorname{Ir}(1)$ & $117.8(2)$ & $\mathrm{C}(15)-\mathrm{C}(3)-\mathrm{P}(1)$ & $119.7(4)$ \\
\hline $\mathrm{C}(28)-\mathrm{P}(2)-\operatorname{Ir}(1)$ & $119.5(2)$ & $\mathrm{C}(4)-\mathrm{C}(3)-\mathrm{P}(1)$ & $110.1(4)$ \\
\hline
\end{tabular}




\begin{tabular}{|c|c|c|c|}
\hline $\mathrm{P}(2)-\mathrm{C}(3)-\mathrm{P}(1)$ & $90.0(3)$ & $C(10)-C(15)-C(3)$ & $109.7(5)$ \\
\hline $\mathrm{C}(5)-\mathrm{C}(4)-\mathrm{C}(9)$ & $120.2(6)$ & $\mathrm{C}(17)-\mathrm{C}(16)-\mathrm{C}(21)$ & $119.1(6)$ \\
\hline$C(5)-C(4)-C(3)$ & $130.2(6)$ & $\mathrm{C}(17)-\mathrm{C}(16)-\mathrm{P}(1)$ & $118.1(5)$ \\
\hline $\mathrm{C}(9)-\mathrm{C}(4)-\mathrm{C}(3)$ & $109.5(5)$ & $\mathrm{C}(21)-\mathrm{C}(16)-\mathrm{P}(1)$ & $122.7(5)$ \\
\hline$C(6)-C(5)-C(4)$ & $118.4(6)$ & $C(16)-C(17)-C(18)$ & $120.4(7)$ \\
\hline $\mathrm{C}(6)-\mathrm{C}(5)-\mathrm{H}(5)$ & 120.8 & $\mathrm{C}(16)-\mathrm{C}(17)-\mathrm{H}(17)$ & 119.8 \\
\hline $\mathrm{C}(4)-\mathrm{C}(5)-\mathrm{H}(5)$ & 120.8 & $\mathrm{C}(18)-\mathrm{C}(17)-\mathrm{H}(17)$ & 119.8 \\
\hline$C(7)-C(6)-C(5)$ & $121.7(6)$ & $\mathrm{C}(19)-\mathrm{C}(18)-\mathrm{C}(17)$ & $119.7(7)$ \\
\hline $\mathrm{C}(7)-\mathrm{C}(6)-\mathrm{H}(6)$ & 119.1 & $\mathrm{C}(19)-\mathrm{C}(18)-\mathrm{H}(18)$ & 120.2 \\
\hline $\mathrm{C}(5)-\mathrm{C}(6)-\mathrm{H}(6)$ & 119.1 & $\mathrm{C}(17)-\mathrm{C}(18)-\mathrm{H}(18)$ & 120.2 \\
\hline $\mathrm{C}(6)-\mathrm{C}(7)-\mathrm{C}(8)$ & $120.5(6)$ & $\mathrm{C}(18)-\mathrm{C}(19)-\mathrm{C}(20)$ & $120.1(7)$ \\
\hline $\mathrm{C}(6)-\mathrm{C}(7)-\mathrm{H}(7)$ & 119.7 & $\mathrm{C}(18)-\mathrm{C}(19)-\mathrm{H}(19)$ & 120.0 \\
\hline $\mathrm{C}(8)-\mathrm{C}(7)-\mathrm{H}(7)$ & 119.7 & $\mathrm{C}(20)-\mathrm{C}(19)-\mathrm{H}(19)$ & 120.0 \\
\hline $\mathrm{C}(9)-\mathrm{C}(8)-\mathrm{C}(7)$ & $118.5(6)$ & $C(21)-C(20)-C(19)$ & $120.5(7)$ \\
\hline $\mathrm{C}(9)-\mathrm{C}(8)-\mathrm{H}(8)$ & 120.7 & $\mathrm{C}(21)-\mathrm{C}(20)-\mathrm{H}(20)$ & 119.8 \\
\hline $\mathrm{C}(7)-\mathrm{C}(8)-\mathrm{H}(8)$ & 120.7 & $\mathrm{C}(19)-\mathrm{C}(20)-\mathrm{H}(20)$ & 119.8 \\
\hline $\mathrm{C}(8)-\mathrm{C}(9)-\mathrm{C}(4)$ & $120.6(6)$ & $C(20)-C(21)-C(16)$ & $120.3(7)$ \\
\hline $\mathrm{C}(8)-\mathrm{C}(9)-\mathrm{C}(10)$ & $130.5(6)$ & $\mathrm{C}(20)-\mathrm{C}(21)-\mathrm{H}(21)$ & 119.9 \\
\hline $\mathrm{C}(4)-\mathrm{C}(9)-\mathrm{C}(10)$ & $108.9(5)$ & $\mathrm{C}(16)-\mathrm{C}(21)-\mathrm{H}(21)$ & 119.9 \\
\hline$C(11)-C(10)-C(15)$ & $121.3(6)$ & $C(23)-C(22)-C(27)$ & $119.5(6)$ \\
\hline $\mathrm{C}(11)-\mathrm{C}(10)-\mathrm{C}(9)$ & $129.7(6)$ & $\mathrm{C}(23)-\mathrm{C}(22)-\mathrm{P}(1)$ & $120.4(5)$ \\
\hline$C(15)-C(10)-C(9)$ & $108.9(5)$ & $\mathrm{C}(27)-\mathrm{C}(22)-\mathrm{P}(1)$ & $120.1(5)$ \\
\hline $\mathrm{C}(10)-\mathrm{C}(11)-\mathrm{C}(12)$ & $118.9(7)$ & $\mathrm{C}(22)-\mathrm{C}(23)-\mathrm{C}(24)$ & $119.4(7)$ \\
\hline $\mathrm{C}(10)-\mathrm{C}(11)-\mathrm{H}(11)$ & 120.5 & $\mathrm{C}(22)-\mathrm{C}(23)-\mathrm{H}(23)$ & 120.3 \\
\hline $\mathrm{C}(12)-\mathrm{C}(11)-\mathrm{H}(11)$ & 120.5 & $\mathrm{C}(24)-\mathrm{C}(23)-\mathrm{H}(23)$ & 120.3 \\
\hline $\mathrm{C}(11)-\mathrm{C}(12)-\mathrm{C}(13)$ & $119.9(6)$ & $\mathrm{C}(25)-\mathrm{C}(24)-\mathrm{C}(23)$ & $120.7(8)$ \\
\hline $\mathrm{C}(11)-\mathrm{C}(12)-\mathrm{H}(12)$ & 120.1 & $\mathrm{C}(25)-\mathrm{C}(24)-\mathrm{H}(24)$ & 119.6 \\
\hline $\mathrm{C}(13)-\mathrm{C}(12)-\mathrm{H}(12)$ & 120.1 & $\mathrm{C}(23)-\mathrm{C}(24)-\mathrm{H}(24)$ & 119.6 \\
\hline $\mathrm{C}(14)-\mathrm{C}(13)-\mathrm{C}(12)$ & $121.7(6)$ & $\mathrm{C}(24)-\mathrm{C}(25)-\mathrm{C}(26)$ & $120.4(6)$ \\
\hline $\mathrm{C}(14)-\mathrm{C}(13)-\mathrm{H}(13)$ & 119.2 & $\mathrm{C}(24)-\mathrm{C}(25)-\mathrm{H}(25)$ & 119.8 \\
\hline $\mathrm{C}(12)-\mathrm{C}(13)-\mathrm{H}(13)$ & 119.2 & $\mathrm{C}(26)-\mathrm{C}(25)-\mathrm{H}(25)$ & 119.8 \\
\hline $\mathrm{C}(13)-\mathrm{C}(14)-\mathrm{C}(15)$ & $118.4(6)$ & $C(25)-C(26)-C(27)$ & $120.2(7)$ \\
\hline $\mathrm{C}(13)-\mathrm{C}(14)-\mathrm{H}(14)$ & 120.8 & $\mathrm{C}(25)-\mathrm{C}(26)-\mathrm{H}(26)$ & 119.9 \\
\hline $\mathrm{C}(15)-\mathrm{C}(14)-\mathrm{H}(14)$ & 120.8 & $\mathrm{C}(27)-\mathrm{C}(26)-\mathrm{H}(26)$ & 119.9 \\
\hline $\mathrm{C}(14)-\mathrm{C}(15)-\mathrm{C}(10)$ & $119.7(6)$ & $\mathrm{C}(26)-\mathrm{C}(27)-\mathrm{C}(22)$ & $119.8(7)$ \\
\hline$C(14)-C(15)-C(3)$ & $130.6(6)$ & $\mathrm{C}(26)-\mathrm{C}(27)-\mathrm{H}(27)$ & 120.1 \\
\hline
\end{tabular}




\begin{tabular}{|c|c|c|c|}
\hline $\mathrm{C}(22)-\mathrm{C}(27)-\mathrm{H}(27)$ & 120.1 & $\mathrm{C}(34)-\mathrm{C}(39)-\mathrm{H}(39)$ & 119.7 \\
\hline$C(29)-C(28)-C(33)$ & $119.7(5)$ & $C(41)-C(40)-C(52)$ & $102.9(6)$ \\
\hline $\mathrm{C}(29)-\mathrm{C}(28)-\mathrm{P}(2)$ & $123.2(5)$ & $\mathrm{C}(41)-\mathrm{C}(40)-\mathrm{P}(4)$ & $112.5(4)$ \\
\hline $\mathrm{C}(33)-\mathrm{C}(28)-\mathrm{P}(2)$ & $117.1(5)$ & $\mathrm{C}(52)-\mathrm{C}(40)-\mathrm{P}(4)$ & $120.0(5)$ \\
\hline $\mathrm{C}(30)-\mathrm{C}(29)-\mathrm{C}(28)$ & $119.7(6)$ & $\mathrm{C}(41)-\mathrm{C}(40)-\mathrm{P}(3)$ & $111.6(5)$ \\
\hline $\mathrm{C}(30)-\mathrm{C}(29)-\mathrm{H}(29)$ & 120.1 & $\mathrm{C}(52)-\mathrm{C}(40)-\mathrm{P}(3)$ & $119.6(4)$ \\
\hline $\mathrm{C}(28)-\mathrm{C}(29)-\mathrm{H}(29)$ & 120.1 & $\mathrm{P}(4)-\mathrm{C}(40)-\mathrm{P}(3)$ & $90.6(3)$ \\
\hline$C(31)-C(30)-C(29)$ & $120.5(7)$ & $\mathrm{C}(42)-\mathrm{C}(41)-\mathrm{C}(46)$ & $120.2(7)$ \\
\hline $\mathrm{C}(31)-\mathrm{C}(30)-\mathrm{H}(30)$ & 119.7 & $C(42)-C(41)-C(40)$ & $129.6(7)$ \\
\hline $\mathrm{C}(29)-\mathrm{C}(30)-\mathrm{H}(30)$ & 119.7 & $\mathrm{C}(46)-\mathrm{C}(41)-\mathrm{C}(40)$ & $110.1(7)$ \\
\hline$C(32)-C(31)-C(30)$ & $119.9(6)$ & $\mathrm{C}(43)-\mathrm{C}(42)-\mathrm{C}(41)$ & $118.1(8)$ \\
\hline $\mathrm{C}(32)-\mathrm{C}(31)-\mathrm{H}(31)$ & 120.0 & $\mathrm{C}(43)-\mathrm{C}(42)-\mathrm{H}(42)$ & 121.0 \\
\hline $\mathrm{C}(30)-\mathrm{C}(31)-\mathrm{H}(31)$ & 120.0 & $\mathrm{C}(41)-\mathrm{C}(42)-\mathrm{H}(42)$ & 121.0 \\
\hline$C(31)-C(32)-C(33)$ & $121.0(6)$ & $\mathrm{C}(44)-\mathrm{C}(43)-\mathrm{C}(42)$ & $121.8(9)$ \\
\hline $\mathrm{C}(31)-\mathrm{C}(32)-\mathrm{H}(32)$ & 119.5 & $\mathrm{C}(44)-\mathrm{C}(43)-\mathrm{H}(43)$ & 119.1 \\
\hline $\mathrm{C}(33)-\mathrm{C}(32)-\mathrm{H}(32)$ & 119.5 & $\mathrm{C}(42)-\mathrm{C}(43)-\mathrm{H}(43)$ & 119.1 \\
\hline$C(32)-C(33)-C(28)$ & 119.2(6) & $C(43)-C(44)-C(45)$ & $121.3(8)$ \\
\hline $\mathrm{C}(32)-\mathrm{C}(33)-\mathrm{H}(33)$ & 120.4 & $\mathrm{C}(43)-\mathrm{C}(44)-\mathrm{H}(44)$ & 119.4 \\
\hline $\mathrm{C}(28)-\mathrm{C}(33)-\mathrm{H}(33)$ & 120.4 & $\mathrm{C}(45)-\mathrm{C}(44)-\mathrm{H}(44)$ & 119.4 \\
\hline $\mathrm{C}(35)-\mathrm{C}(34)-\mathrm{C}(39)$ & $117.8(6)$ & $\mathrm{C}(44)-\mathrm{C}(45)-\mathrm{C}(46)$ & $118.6(8)$ \\
\hline $\mathrm{C}(35)-\mathrm{C}(34)-\mathrm{P}(2)$ & $120.6(4)$ & $\mathrm{C}(44)-\mathrm{C}(45)-\mathrm{H}(45)$ & 120.7 \\
\hline $\mathrm{C}(39)-\mathrm{C}(34)-\mathrm{P}(2)$ & $121.4(5)$ & $\mathrm{C}(46)-\mathrm{C}(45)-\mathrm{H}(45)$ & 120.7 \\
\hline$C(36)-C(35)-C(34)$ & $120.2(6)$ & $\mathrm{C}(45)-\mathrm{C}(46)-\mathrm{C}(41)$ & $120.1(8)$ \\
\hline $\mathrm{C}(36)-\mathrm{C}(35)-\mathrm{H}(35)$ & 119.9 & $\mathrm{C}(45)-\mathrm{C}(46)-\mathrm{C}(47)$ & $132.0(8)$ \\
\hline $\mathrm{C}(34)-\mathrm{C}(35)-\mathrm{H}(35)$ & 119.9 & $\mathrm{C}(41)-\mathrm{C}(46)-\mathrm{C}(47)$ & $107.9(7)$ \\
\hline$C(37)-C(36)-C(35)$ & $120.2(7)$ & $\mathrm{C}(48)-\mathrm{C}(47)-\mathrm{C}(52)$ & $120.3(9)$ \\
\hline $\mathrm{C}(37)-\mathrm{C}(36)-\mathrm{H}(36)$ & 119.9 & $\mathrm{C}(48)-\mathrm{C}(47)-\mathrm{C}(46)$ & $130.5(9)$ \\
\hline $\mathrm{C}(35)-\mathrm{C}(36)-\mathrm{H}(36)$ & 119.9 & $C(52)-C(47)-C(46)$ & $109.2(6)$ \\
\hline $\mathrm{C}(38)-\mathrm{C}(37)-\mathrm{C}(36)$ & $119.6(7)$ & $\mathrm{C}(49)-\mathrm{C}(48)-\mathrm{C}(47)$ & $119.3(10)$ \\
\hline $\mathrm{C}(38)-\mathrm{C}(37)-\mathrm{H}(37)$ & 120.2 & $\mathrm{C}(49)-\mathrm{C}(48)-\mathrm{H}(48)$ & 120.3 \\
\hline $\mathrm{C}(36)-\mathrm{C}(37)-\mathrm{H}(37)$ & 120.2 & $\mathrm{C}(47)-\mathrm{C}(48)-\mathrm{H}(48)$ & 120.3 \\
\hline $\mathrm{C}(37)-\mathrm{C}(38)-\mathrm{C}(39)$ & $121.5(6)$ & $\mathrm{C}(48)-\mathrm{C}(49)-\mathrm{C}(50)$ & $121.6(8)$ \\
\hline $\mathrm{C}(37)-\mathrm{C}(38)-\mathrm{H}(38)$ & 119.3 & $\mathrm{C}(48)-\mathrm{C}(49)-\mathrm{H}(49)$ & 119.2 \\
\hline $\mathrm{C}(39)-\mathrm{C}(38)-\mathrm{H}(38)$ & 119.3 & $\mathrm{C}(50)-\mathrm{C}(49)-\mathrm{H}(49)$ & 119.2 \\
\hline $\mathrm{C}(38)-\mathrm{C}(39)-\mathrm{C}(34)$ & $120.7(6)$ & $\mathrm{C}(51)-\mathrm{C}(50)-\mathrm{C}(49)$ & 119.1(10) \\
\hline $\mathrm{C}(38)-\mathrm{C}(39)-\mathrm{H}(39)$ & 119.7 & $\mathrm{C}(51)-\mathrm{C}(50)-\mathrm{H}(50)$ & 120.4 \\
\hline
\end{tabular}




\begin{tabular}{|c|c|c|c|}
\hline $\mathrm{C}(49)-\mathrm{C}(50)-\mathrm{H}(50)$ & 120.4 & $\mathrm{C}(61)-\mathrm{C}(62)-\mathrm{H}(62)$ & 120.1 \\
\hline $\mathrm{C}(52)-\mathrm{C}(51)-\mathrm{C}(50)$ & $120.0(9)$ & $\mathrm{C}(62)-\mathrm{C}(63)-\mathrm{C}(64)$ & 121.1(8) \\
\hline $\mathrm{C}(52)-\mathrm{C}(51)-\mathrm{H}(51)$ & 120.0 & $\mathrm{C}(62)-\mathrm{C}(63)-\mathrm{H}(63)$ & 119.5 \\
\hline $\mathrm{C}(50)-\mathrm{C}(51)-\mathrm{H}(51)$ & 120.0 & $\mathrm{C}(64)-\mathrm{C}(63)-\mathrm{H}(63)$ & 119.5 \\
\hline $\mathrm{C}(51)-\mathrm{C}(52)-\mathrm{C}(47)$ & 119.7(7) & $\mathrm{C}(63)-\mathrm{C}(64)-\mathrm{C}(59)$ & $120.1(8)$ \\
\hline$C(51)-C(52)-C(40)$ & $130.5(7)$ & $\mathrm{C}(63)-\mathrm{C}(64)-\mathrm{H}(64)$ & 120.0 \\
\hline$C(47)-C(52)-C(40)$ & $109.8(7)$ & $\mathrm{C}(59)-\mathrm{C}(64)-\mathrm{H}(64)$ & 120.0 \\
\hline $\mathrm{C}(54)-\mathrm{C}(53)-\mathrm{C}(58)$ & $118.9(7)$ & $\mathrm{C}(70)-\mathrm{C}(65)-\mathrm{C}(66)$ & $119.4(6)$ \\
\hline $\mathrm{C}(54)-\mathrm{C}(53)-\mathrm{P}(3)$ & $117.7(5)$ & $\mathrm{C}(70)-\mathrm{C}(65)-\mathrm{P}(4)$ & $116.8(5)$ \\
\hline $\mathrm{C}(58)-\mathrm{C}(53)-\mathrm{P}(3)$ & $123.3(6)$ & $\mathrm{C}(66)-\mathrm{C}(65)-\mathrm{P}(4)$ & $123.5(5)$ \\
\hline C(53)-C(54)-C(55) & $121.0(9)$ & $\mathrm{C}(67)-\mathrm{C}(66)-\mathrm{C}(65)$ & $119.3(7)$ \\
\hline $\mathrm{C}(53)-\mathrm{C}(54)-\mathrm{H}(54)$ & 119.5 & $\mathrm{C}(67)-\mathrm{C}(66)-\mathrm{H}(66)$ & 120.4 \\
\hline $\mathrm{C}(55)-\mathrm{C}(54)-\mathrm{H}(54)$ & 119.5 & $\mathrm{C}(65)-\mathrm{C}(66)-\mathrm{H}(66)$ & 120.4 \\
\hline$C(56)-C(55)-C(54)$ & $118.6(10)$ & $C(68)-C(67)-C(66)$ & $120.4(7)$ \\
\hline $\mathrm{C}(56)-\mathrm{C}(55)-\mathrm{H}(84)$ & 120.7 & $\mathrm{C}(68)-\mathrm{C}(67)-\mathrm{H}(67)$ & 119.8 \\
\hline $\mathrm{C}(54)-\mathrm{C}(55)-\mathrm{H}(84)$ & 120.7 & $\mathrm{C}(66)-\mathrm{C}(67)-\mathrm{H}(67)$ & 119.8 \\
\hline$C(55)-C(56)-C(57)$ & $121.0(8)$ & $\mathrm{C}(69)-\mathrm{C}(68)-\mathrm{C}(67)$ & $120.4(6)$ \\
\hline $\mathrm{C}(55)-\mathrm{C}(56)-\mathrm{H}(56)$ & 119.5 & $\mathrm{C}(69)-\mathrm{C}(68)-\mathrm{H}(68)$ & 119.8 \\
\hline $\mathrm{C}(57)-\mathrm{C}(56)-\mathrm{H}(56)$ & 119.5 & $\mathrm{C}(67)-\mathrm{C}(68)-\mathrm{H}(68)$ & 119.8 \\
\hline $\mathrm{C}(58)-\mathrm{C}(57)-\mathrm{C}(56)$ & 119.0(9) & $\mathrm{C}(68)-\mathrm{C}(69)-\mathrm{C}(70)$ & $119.7(7)$ \\
\hline $\mathrm{C}(58)-\mathrm{C}(57)-\mathrm{H}(57)$ & 120.5 & $\mathrm{C}(68)-\mathrm{C}(69)-\mathrm{H}(69)$ & 120.2 \\
\hline $\mathrm{C}(56)-\mathrm{C}(57)-\mathrm{H}(57)$ & 120.5 & $\mathrm{C}(70)-\mathrm{C}(69)-\mathrm{H}(69)$ & 120.2 \\
\hline $\mathrm{C}(57)-\mathrm{C}(58)-\mathrm{C}(53)$ & $121.5(9)$ & $\mathrm{C}(65)-\mathrm{C}(70)-\mathrm{C}(69)$ & $120.8(7)$ \\
\hline $\mathrm{C}(57)-\mathrm{C}(58)-\mathrm{H}(58)$ & 119.3 & $\mathrm{C}(65)-\mathrm{C}(70)-\mathrm{H}(70)$ & 119.6 \\
\hline $\mathrm{C}(53)-\mathrm{C}(58)-\mathrm{H}(58)$ & 119.3 & $\mathrm{C}(69)-\mathrm{C}(70)-\mathrm{H}(70)$ & 119.6 \\
\hline$C(60)-C(59)-C(64)$ & $117.8(6)$ & $C(72)-C(71)-C(76)$ & $119.0(7)$ \\
\hline $\mathrm{C}(60)-\mathrm{C}(59)-\mathrm{P}(3)$ & $121.3(5)$ & $\mathrm{C}(72)-\mathrm{C}(71)-\mathrm{P}(4)$ & $120.3(6)$ \\
\hline $\mathrm{C}(64)-\mathrm{C}(59)-\mathrm{P}(3)$ & $120.8(6)$ & $\mathrm{C}(76)-\mathrm{C}(71)-\mathrm{P}(4)$ & $120.6(5)$ \\
\hline $\mathrm{C}(59)-\mathrm{C}(60)-\mathrm{C}(61)$ & $121.6(7)$ & $\mathrm{C}(73)-\mathrm{C}(72)-\mathrm{C}(71)$ & $120.9(7)$ \\
\hline $\mathrm{C}(59)-\mathrm{C}(60)-\mathrm{H}(60)$ & 119.2 & $\mathrm{C}(73)-\mathrm{C}(72)-\mathrm{H}(72)$ & 119.5 \\
\hline $\mathrm{C}(61)-\mathrm{C}(60)-\mathrm{H}(60)$ & 119.2 & $\mathrm{C}(71)-\mathrm{C}(72)-\mathrm{H}(72)$ & 119.5 \\
\hline $\mathrm{C}(62)-\mathrm{C}(61)-\mathrm{C}(60)$ & 119.5(9) & $\mathrm{C}(72)-\mathrm{C}(73)-\mathrm{C}(74)$ & $120.7(8)$ \\
\hline $\mathrm{C}(62)-\mathrm{C}(61)-\mathrm{H}(61)$ & 120.3 & $\mathrm{C}(72)-\mathrm{C}(73)-\mathrm{H}(73)$ & 119.7 \\
\hline $\mathrm{C}(60)-\mathrm{C}(61)-\mathrm{H}(61)$ & 120.3 & $\mathrm{C}(74)-\mathrm{C}(73)-\mathrm{H}(73)$ & 119.7 \\
\hline $\mathrm{C}(63)-\mathrm{C}(62)-\mathrm{C}(61)$ & 119.9(7) & $\mathrm{C}(75)-\mathrm{C}(74)-\mathrm{C}(73)$ & $118.9(7)$ \\
\hline $\mathrm{C}(63)-\mathrm{C}(62)-\mathrm{H}(62)$ & 120.1 & $\mathrm{C}(75)-\mathrm{C}(74)-\mathrm{H}(74)$ & 120.5 \\
\hline
\end{tabular}




\begin{tabular}{|c|c|c|c|}
\hline $\mathrm{C}(73)-\mathrm{C}(74)-\mathrm{H}(74)$ & 120.5 & $\mathrm{H}(81)-\mathrm{C}(81)-\mathrm{H}(81 \mathrm{~B})$ & 109.5 \\
\hline $\mathrm{C}(74)-\mathrm{C}(75)-\mathrm{C}(76)$ & $121.0(7)$ & $\mathrm{H}(81 \mathrm{~A})-\mathrm{C}(81)-\mathrm{H}(81 \mathrm{~B})$ & 109.5 \\
\hline $\mathrm{C}(74)-\mathrm{C}(75)-\mathrm{H}(75)$ & 119.5 & $\mathrm{O}(5)-\mathrm{C}(82)-\mathrm{C}(83)$ & $121.2(10)$ \\
\hline $\mathrm{C}(76)-\mathrm{C}(75)-\mathrm{H}(75)$ & 119.5 & $\mathrm{O}(5)-\mathrm{C}(82)-\mathrm{C}(81)$ & $120.8(9)$ \\
\hline$C(71)-C(76)-C(75)$ & $119.5(7)$ & $\mathrm{C}(83)-\mathrm{C}(82)-\mathrm{C}(81)$ & $117.8(10)$ \\
\hline $\mathrm{C}(71)-\mathrm{C}(76)-\mathrm{H}(76)$ & 120.3 & $\mathrm{C}(82)-\mathrm{C}(83)-\mathrm{H}(83)$ & 109.5 \\
\hline $\mathrm{C}(75)-\mathrm{C}(76)-\mathrm{H}(76)$ & 120.3 & $\mathrm{C}(82)-\mathrm{C}(83)-\mathrm{H}(83 \mathrm{~A})$ & 109.5 \\
\hline C(78)-C(77)-H(77) & 109.5 & $\mathrm{H}(83)-\mathrm{C}(83)-\mathrm{H}(83 \mathrm{~A})$ & 109.5 \\
\hline $\mathrm{C}(78)-\mathrm{C}(77)-\mathrm{H}(77 \mathrm{~A})$ & 109.5 & $\mathrm{C}(82)-\mathrm{C}(83)-\mathrm{H}(83 \mathrm{~B})$ & 109.5 \\
\hline H(77)-C(77)-H(77A) & 109.5 & $\mathrm{H}(83)-\mathrm{C}(83)-\mathrm{H}(83 \mathrm{~B})$ & 109.5 \\
\hline $\mathrm{C}(78)-\mathrm{C}(77)-\mathrm{H}(77 \mathrm{~B})$ & 109.5 & $\mathrm{H}(83 \mathrm{~A})-\mathrm{C}(83)-\mathrm{H}(83 \mathrm{~B})$ & 109.5 \\
\hline H(77)-C(77)-H(77B) & 109.5 & $\mathrm{~F}(5)-\mathrm{B}(1)-\mathrm{F}(9)$ & $123.8(13)$ \\
\hline H(77A)-C(77)-H(77B) & 109.5 & $\mathrm{~F}(5)-\mathrm{B}(1)-\mathrm{F}(7)$ & $65.1(16)$ \\
\hline $\mathrm{O}(4)-\mathrm{C}(78)-\mathrm{C}(77)$ & $109.6(8)$ & $\mathrm{F}(9)-\mathrm{B}(1)-\mathrm{F}(7)$ & $68.6(15)$ \\
\hline $\mathrm{O}(4)-\mathrm{C}(78)-\mathrm{H}(78)$ & 109.7 & $F(5)-B(1)-F(4)$ & $45.4(10)$ \\
\hline $\mathrm{C}(77)-\mathrm{C}(78)-\mathrm{H}(78)$ & 109.7 & $\mathrm{~F}(9)-\mathrm{B}(1)-\mathrm{F}(4)$ & $132.0(13)$ \\
\hline $\mathrm{O}(4)-\mathrm{C}(78)-\mathrm{H}(78 \mathrm{~A})$ & 109.7 & $\mathrm{~F}(7)-\mathrm{B}(1)-\mathrm{F}(4)$ & $107.0(16)$ \\
\hline $\mathrm{C}(77)-\mathrm{C}(78)-\mathrm{H}(78 \mathrm{~A})$ & 109.7 & $\mathrm{~F}(5)-\mathrm{B}(1)-\mathrm{F}(1)$ & $114.1(10)$ \\
\hline $\mathrm{H}(78)-\mathrm{C}(78)-\mathrm{H}(78 \mathrm{~A})$ & 108.2 & $\mathrm{~F}(9)-\mathrm{B}(1)-\mathrm{F}(1)$ & $113.8(10)$ \\
\hline $\mathrm{C}(79)-\mathrm{O}(4)-\mathrm{C}(78)$ & $109.7(8)$ & $\mathrm{F}(7)-\mathrm{B}(1)-\mathrm{F}(1)$ & $116.1(14)$ \\
\hline $\mathrm{O}(4)-\mathrm{C}(79)-\mathrm{C}(80)$ & 109.1(9) & $\mathrm{F}(4)-\mathrm{B}(1)-\mathrm{F}(1)$ & $110.5(9)$ \\
\hline $\mathrm{O}(4)-\mathrm{C}(79)-\mathrm{H}(79)$ & 109.9 & $\mathrm{~F}(5)-\mathrm{B}(1)-\mathrm{F}(2)$ & $116.0(16)$ \\
\hline $\mathrm{C}(80)-\mathrm{C}(79)-\mathrm{H}(79)$ & 109.9 & $\mathrm{~F}(9)-\mathrm{B}(1)-\mathrm{F}(2)$ & $77.3(15)$ \\
\hline $\mathrm{O}(4)-\mathrm{C}(79)-\mathrm{H}(79 \mathrm{~A})$ & 109.9 & $\mathrm{~F}(7)-\mathrm{B}(1)-\mathrm{F}(2)$ & $134.5(18)$ \\
\hline $\mathrm{C}(80)-\mathrm{C}(79)-\mathrm{H}(79 \mathrm{~A})$ & 109.9 & $\mathrm{~F}(4)-\mathrm{B}(1)-\mathrm{F}(2)$ & $74.6(14)$ \\
\hline H(79)-C(79)-H(79A) & 108.3 & $\mathrm{~F}(1)-\mathrm{B}(1)-\mathrm{F}(2)$ & $104.7(13)$ \\
\hline $\mathrm{C}(79)-\mathrm{C}(80)-\mathrm{H}(80)$ & 109.5 & $\mathrm{~F}(5)-\mathrm{B}(1)-\mathrm{F}(10)$ & $134.3(12)$ \\
\hline $\mathrm{C}(79)-\mathrm{C}(80)-\mathrm{H}(80 \mathrm{~A})$ & 109.5 & $\mathrm{~F}(9)-\mathrm{B}(1)-\mathrm{F}(10)$ & $39.6(9)$ \\
\hline $\mathrm{H}(80)-\mathrm{C}(80)-\mathrm{H}(80 \mathrm{~A})$ & 109.5 & $\mathrm{~F}(7)-\mathrm{B}(1)-\mathrm{F}(10)$ & $105.4(15)$ \\
\hline $\mathrm{C}(79)-\mathrm{C}(80)-\mathrm{H}(80 \mathrm{~B})$ & 109.5 & $\mathrm{~F}(4)-\mathrm{B}(1)-\mathrm{F}(10)$ & $107.5(12)$ \\
\hline $\mathrm{H}(80)-\mathrm{C}(80)-\mathrm{H}(80 \mathrm{~B})$ & 109.5 & $\mathrm{~F}(1)-\mathrm{B}(1)-\mathrm{F}(10)$ & $110.0(9)$ \\
\hline $\mathrm{H}(80 \mathrm{~A})-\mathrm{C}(80)-\mathrm{H}(80 \mathrm{~B})$ & 109.5 & $\mathrm{~F}(2)-\mathrm{B}(1)-\mathrm{F}(10)$ & $38.2(11)$ \\
\hline $\mathrm{C}(82)-\mathrm{C}(81)-\mathrm{H}(81)$ & 109.5 & $\mathrm{~F}(5)-\mathrm{B}(1)-\mathrm{F}(8)$ & $108.4(12)$ \\
\hline $\mathrm{C}(82)-\mathrm{C}(81)-\mathrm{H}(81 \mathrm{~A})$ & 109.5 & $\mathrm{~F}(9)-\mathrm{B}(1)-\mathrm{F}(8)$ & $29.5(8)$ \\
\hline $\mathrm{H}(81)-\mathrm{C}(81)-\mathrm{H}(81 \mathrm{~A})$ & 109.5 & $\mathrm{~F}(7)-\mathrm{B}(1)-\mathrm{F}(8)$ & $44.2(13)$ \\
\hline C(82)-C(81)-H(81B) & 109.5 & $\mathrm{~F}(4)-\mathrm{B}(1)-\mathrm{F}(8)$ & $141.6(11)$ \\
\hline
\end{tabular}




\begin{tabular}{|c|c|c|c|}
\hline $\mathrm{F}(1)-\mathrm{B}(1)-\mathrm{F}(8)$ & 106.2(9) & $F(3)-F(4)-F(5)$ & $145(3)$ \\
\hline $\mathrm{F}(2)-\mathrm{B}(1)-\mathrm{F}(8)$ & $106.8(14)$ & $\mathrm{F}(3)-\mathrm{F}(4)-\mathrm{B}(1)$ & $87(3)$ \\
\hline $\mathrm{F}(10)-\mathrm{B}(1)-\mathrm{F}(8)$ & $69.0(9)$ & $\mathrm{F}(5)-\mathrm{F}(4)-\mathrm{B}(1)$ & $61.0(13)$ \\
\hline $\mathrm{F}(5)-\mathrm{B}(1)-\mathrm{F}(3)$ & $70.5(13)$ & $\mathrm{F}(3)-\mathrm{F}(4)-\mathrm{F}(2)$ & $34(3)$ \\
\hline $\mathrm{F}(9)-\mathrm{B}(1)-\mathrm{F}(3)$ & $118.2(14)$ & $F(5)-F(4)-F(2)$ & $110.4(17)$ \\
\hline $\mathrm{F}(7)-\mathrm{B}(1)-\mathrm{F}(3)$ & $126.2(17)$ & $\mathrm{B}(1)-\mathrm{F}(4)-\mathrm{F}(2)$ & $53.3(11)$ \\
\hline $\mathrm{F}(4)-\mathrm{B}(1)-\mathrm{F}(3)$ & $25.7(9)$ & $F(3)-F(4)-F(6)$ & $140(3)$ \\
\hline $\mathrm{F}(1)-\mathrm{B}(1)-\mathrm{F}(3)$ & $108.6(10)$ & $F(5)-F(4)-F(6)$ & $5.8(13)$ \\
\hline $\mathrm{F}(2)-\mathrm{B}(1)-\mathrm{F}(3)$ & $49.4(13)$ & $\mathrm{B}(1)-\mathrm{F}(4)-\mathrm{F}(6)$ & $55.4(10)$ \\
\hline $\mathrm{F}(10)-\mathrm{B}(1)-\mathrm{F}(3)$ & $84.9(12)$ & $\mathrm{F}(2)-\mathrm{F}(4)-\mathrm{F}(6)$ & $105.6(14)$ \\
\hline $\mathrm{F}(8)-\mathrm{B}(1)-\mathrm{F}(3)$ & $141.9(12)$ & $F(6)-F(5)-F(4)$ & $166(3)$ \\
\hline $\mathrm{F}(5)-\mathrm{B}(1)-\mathrm{F}(6)$ & $30.2(10)$ & $\mathrm{F}(6)-\mathrm{F}(5)-\mathrm{B}(1)$ & $93(3)$ \\
\hline $\mathrm{F}(9)-\mathrm{B}(1)-\mathrm{F}(6)$ & $107.9(14)$ & $\mathrm{F}(4)-\mathrm{F}(5)-\mathrm{B}(1)$ & $73.6(15)$ \\
\hline $\mathrm{F}(7)-\mathrm{B}(1)-\mathrm{F}(6)$ & $39.9(13)$ & $F(6)-F(5)-F(7)$ & $43(2)$ \\
\hline $\mathrm{F}(4)-\mathrm{B}(1)-\mathrm{F}(6)$ & $75.6(12)$ & $\mathrm{F}(4)-\mathrm{F}(5)-\mathrm{F}(7)$ & $127(2)$ \\
\hline $\mathrm{F}(1)-\mathrm{B}(1)-\mathrm{F}(6)$ & $106.3(11)$ & $\mathrm{B}(1)-\mathrm{F}(5)-\mathrm{F}(7)$ & $59.5(14)$ \\
\hline $\mathrm{F}(2)-\mathrm{B}(1)-\mathrm{F}(6)$ & $142.8(16)$ & $\mathrm{F}(6)-\mathrm{F}(5)-\mathrm{F}(3)$ & $155(3)$ \\
\hline $\mathrm{F}(10)-\mathrm{B}(1)-\mathrm{F}(6)$ & $139.3(13)$ & $F(4)-F(5)-F(3)$ & $13.5(13)$ \\
\hline $\mathrm{F}(8)-\mathrm{B}(1)-\mathrm{F}(6)$ & $83.8(12)$ & $\mathrm{B}(1)-\mathrm{F}(5)-\mathrm{F}(3)$ & $61.5(12)$ \\
\hline $\mathrm{F}(3)-\mathrm{B}(1)-\mathrm{F}(6)$ & $100.7(14)$ & $F(7)-F(5)-F(3)$ & $114.0(18)$ \\
\hline$F(10)-F(2)-F(3)$ & $135(3)$ & $F(5)-F(6)-F(7)$ & $105(3)$ \\
\hline$F(10)-F(2)-B(1)$ & $73(2)$ & $\mathrm{F}(5)-\mathrm{F}(6)-\mathrm{B}(1)$ & $57(2)$ \\
\hline $\mathrm{F}(3)-\mathrm{F}(2)-\mathrm{B}(1)$ & $69.1(17)$ & $F(7)-F(6)-B(1)$ & $60(2)$ \\
\hline$F(10)-F(2)-F(4)$ & $118(3)$ & $F(5)-F(6)-F(4)$ & $7.8(18)$ \\
\hline$F(3)-F(2)-F(4)$ & $17.6(13)$ & $F(7)-F(6)-F(4)$ & $100(3)$ \\
\hline$B(1)-F(2)-F(4)$ & $52.1(11)$ & $\mathrm{B}(1)-\mathrm{F}(6)-\mathrm{F}(4)$ & $49.0(10)$ \\
\hline$F(10)-F(2)-F(9)$ & $25.5(14)$ & $F(6)-F(7)-F(8)$ & $154(4)$ \\
\hline$F(3)-F(2)-F(9)$ & $110(2)$ & $F(6)-F(7)-B(1)$ & $81(3)$ \\
\hline $\mathrm{B}(1)-\mathrm{F}(2)-\mathrm{F}(9)$ & $48.8(11)$ & $\mathrm{F}(8)-\mathrm{F}(7)-\mathrm{B}(1)$ & $74.8(19)$ \\
\hline $\mathrm{F}(4)-\mathrm{F}(2)-\mathrm{F}(9)$ & $93.2(15)$ & $F(6)-F(7)-F(5)$ & $31.9(18)$ \\
\hline$F(4)-F(3)-F(2)$ & $128(4)$ & $\mathrm{F}(8)-\mathrm{F}(7)-\mathrm{F}(5)$ & $129(3)$ \\
\hline $\mathrm{F}(4)-\mathrm{F}(3)-\mathrm{B}(1)$ & $68(3)$ & $\mathrm{B}(1)-\mathrm{F}(7)-\mathrm{F}(5)$ & $55.4(14)$ \\
\hline$F(2)-F(3)-B(1)$ & $61.6(16)$ & $F(6)-F(7)-F(9)$ & $135(3)$ \\
\hline$F(4)-F(3)-F(5)$ & $22(2)$ & $F(8)-F(7)-F(9)$ & $26.6(12)$ \\
\hline$F(2)-F(3)-F(5)$ & $106(2)$ & $\mathrm{B}(1)-\mathrm{F}(7)-\mathrm{F}(9)$ & $55.4(13)$ \\
\hline$B(1)-F(3)-F(5)$ & $48.0(10)$ & $F(5)-F(7)-F(9)$ & $104(2)$ \\
\hline
\end{tabular}




$\begin{array}{lclr}F(9)-F(8)-F(7) & 113(3) & F(10)-F(9)-F(2) & 25.2(13) \\ F(9)-F(8)-B(1) & 64.0(18) & B(1)-F(9)-F(2) & 53.9(11) \\ F(7)-F(8)-B(1) & 61.1(17) & F(7)-F(9)-F(2) & 104.2(16) \\ F(9)-F(8)-F(10) & 9.4(16) & F(2)-F(10)-F(9) & 129(3) \\ F(7)-F(8)-F(10) & 107.6(19) & F(2)-F(10)-B(1) & 68.4(19) \\ B(1)-F(8)-F(10) & 55.3(8) & F(9)-F(10)-B(1) & 62.3(13) \\ F(8)-F(9)-F(10) & 163(3) & F(2)-F(10)-F(8) & 123(2) \\ F(8)-F(9)-B(1) & 86(2) & F(9)-F(10)-F(8) & 7.1(12) \\ F(10)-F(9)-B(1) & 78.1(15) & B(1)-F(10)-F(8) & 55.7(8) \\ F(8)-F(9)-F(7) & 40.9(18) & & \\ F(10)-F(9)-F(7) & 129(2) & \text { Symmetry transformations used to generate } \\ B(1)-F(9)-F(7) & 56.0(13) & 140(2) & \end{array}$

(i) Frisch, M. J.; Trucks, G. W.; Schlegel, H. B.; Scuseria, G. E.; Robb, M. A.; Cheeseman, J. R.; Scalmani, G.; Barone, V.; Petersson, G. A.; Nakatsuji, H.; Li, X.; Caricato, M.; Marenich, A. V.; Bloino, J.; Janesko, B. G.; Gomperts, R.; Mennucci, B.; Hratchian, H. P.; Ortiz, J. V.; Izmaylov, A. F.; Sonnenberg, J. L.; Williams-Young, D.; Ding, F.; Lipparini, F.; Egidi, F.; Goings, J.; Peng, B.; Petrone, A.; Henderson, T.; Ranasinghe, D.; Zakrzewski, V. G.; Gao, J.; Rega, N.; Zheng, G.; Liang, W.; Hada, M.; Ehara, M.; Toyota, K.; Fukuda, R.; Hasegawa, J.; Ishida, M.; Nakajima, T.; Honda, Y.; Kitao, O.; Nakai, H.; Vreven, T.; Throssell, K.; Montgomery, J. A., Jr.; Peralta, J. E.; Ogliaro, F.; Bearpark, M. J.; Heyd, J. J.; Brothers, E. N.; Kudin, K. N.; Staroverov, V. N.; Keith, T. A.; Kobayashi, R.; Normand, J.; Raghavachari, K.; Rendell, A. P.; Burant, J. C.; Iyengar, S. S.; Tomasi, J.; Cossi, M.; Millam, J. M.; Klene, M.; Adamo, C.; Cammi, R.; Ochterski, J. W.; Martin, R. L.; Morokuma, K.; Farkas, O.; Foresman, J. B.; Fox D. J. Gaussian 16, Revision A.03, Gaussian, Inc., Wallingford, CT, 2016.

(ii)(a) Becke, A. D. Density-Functional Exchange-Energy Approximation with Correct Asymptotic Behavior. Phys. Rev. A 1988, 38, 3098-3100; (b) Becke, A. D. DensityFunctional Thermochemistry. III. The Role of Exact Exchange. J. Chem. Phys. 1993, 98, 5648-5652; (c) Lee, C.; Yang, W.; Parr, R. G. Development of the Colle-Salvetti Correlation-Energy Formula into a Functional of the Electron Density. Phys. Rev. B 1988, 37, 785-789.

(iii)(a) Dunning, T. H., Jr.; Hay, P. J. In Modern Theoretical Chemistry, Schaefer, H. F., III, Ed.; Plenum: New York, 1976; Vol. 3, pp 1-28; (b) Hay, P. J.; Wadt, W. R. Ab Initio Effective Core Potentials for Molecular Calculations. Potentials for the Transition Metal Atoms Sc to Hg. J. Chem. Phys. 1985, 82, 270-283; (c) Hay, P. J.; Wadt, W. R. Ab Initio Effective Core Potentials for Molecular Calculations. Potentials for $\mathrm{K}$ to Au Including the Outermost Core Orbitals. J. Chem. Phys. 1985, 82, 299-310; (d) Wadt, W. R.; Hay, P. J. $A b$ Initio Effective Core Potentials for Molecular Calculations. Potentials for Main Group Elements $\mathrm{Na}$ to Bi. J. Chem. Phys. 1985, 82, 284-298.

(iv) Petersson, G. A.; Bennett, A.; Tensfeldt, T. G.; Al-Laham, M. A.; Shirley, W. A.; Mantzaris, J. A Complete Basis Set Model Chemistry. I. The Total Energies of Closed-Shell Atoms and Hydrides of the First-Row Elements. J. Chem. Phys. 1988, 89, 2193-2218.

(v) Binkley, J. S.; Pople, J. A.; Hehre, W. J. Self-Consistent Molecular Orbital Methods. 21. 
Small Split-Valence Basis Sets for First-Row Elements. J. Am. Chem. Soc. 1980, 102, 939-947.

(vi) Frisch, M. J.; Trucks, G. W.; Schlegel, H. B.; Scuseria, G. E.; Robb, M. A.; Cheeseman, J. R.; Scalmani, G.; Barone, V.; Petersson, G. A.; Nakatsuji, H.; Li, X.; Caricato, M.; Marenich, A. V.; Bloino, J.; Janesko, B. G.; Gomperts, R.; Mennucci, B.; Hratchian, H. P.; Ortiz, J. V.; Izmaylov, A. F.; Sonnenberg, J. L.; Williams-Young, D.; Ding, F.; Lipparini, F.; Egidi, F.; Goings, J.; Peng, B.; Petrone, A.; Henderson, T.; Ranasinghe, D.; Zakrzewski, V. G.; Gao, J.; Rega, N.; Zheng, G.; Liang, W.; Hada, M.; Ehara, M.; Toyota, K.; Fukuda, R.; Hasegawa, J.; Ishida, M.; Nakajima, T.; Honda, Y.; Kitao, O.; Nakai, H.; Vreven, T.; Throssell, K.; Montgomery, J. A., Jr.; Peralta, J. E.; Ogliaro, F.; Bearpark, M. J.; Heyd, J. J.; Brothers, E. N.; Kudin, K. N.; Staroverov, V. N.; Keith, T. A.; Kobayashi, R.; Normand, J.; Raghavachari, K.; Rendell, A. P.; Burant, J. C.; lyengar, S. S.; Tomasi, J.; Cossi, M.; Millam, J. M.; Klene, M.; Adamo, C.; Cammi, R.; Ochterski, J. W.; Martin, R. L.; Morokuma, K.; Farkas, O.; Foresman, J. B.; Fox D. J. Gaussian 16, Revision A.03, Gaussian, Inc., Wallingford, CT, 2016.

(vii)(a) Becke, A. D. Density-Functional Exchange-Energy Approximation with Correct Asymptotic Behavior. Phys. Rev. A 1988, 38, 3098-3100; (b) Becke, A. D. DensityFunctional Thermochemistry. III. The Role of Exact Exchange. J. Chem. Phys. 1993, 98, 5648-5652; (c) Lee, C.; Yang, W.; Parr, R. G. Development of the Colle-Salvetti Correlation-Energy Formula into a Functional of the Electron Density. Phys. Rev. B 1988, 37, 785-789.

(viii)(a) Dunning, T. H., Jr.; Hay, P. J. In Modern Theoretical Chemistry, Schaefer, H. F., III, Ed.; Plenum: New York, 1976; Vol. 3, pp 1-28; (b) Hay, P. J.; Wadt, W. R. Ab Initio Effective Core Potentials for Molecular Calculations. Potentials for the Transition Metal Atoms Sc to Hg. J. Chem. Phys. 1985, 82, 270-283; (c) Hay, P. J.; Wadt, W. R. Ab Initio Effective Core Potentials for Molecular Calculations. Potentials for $\mathrm{K}$ to Au Including the Outermost Core Orbitals. J. Chem. Phys. 1985, 82, 299-310; (d) Wadt, W. R.; Hay, P. J. $A b$ Initio Effective Core Potentials for Molecular Calculations. Potentials for Main Group Elements $\mathrm{Na}$ to Bi. J. Chem. Phys. 1985, 82, 284-298. 